\title{
Complexation Behavior of Polyelectrolytes and Polyampholytes
}

Arun Kumar Narayanan Nair, Arturo Martinez Jimenez, and Shuyu Sun

J. Phys. Chem. B, Just Accepted Manuscript • DOI: 10.1021/acs.jpcb.7b04582 • Publication Date (Web): 25 Jul 2017

Downloaded from http://pubs.acs.org on July 30, 2017

\section{Just Accepted}

"Just Accepted" manuscripts have been peer-reviewed and accepted for publication. They are posted online prior to technical editing, formatting for publication and author proofing. The American Chemical Society provides "Just Accepted" as a free service to the research community to expedite the dissemination of scientific material as soon as possible after acceptance. "Just Accepted" manuscripts appear in full in PDF format accompanied by an HTML abstract. "Just Accepted" manuscripts have been fully peer reviewed, but should not be considered the official version of record. They are accessible to all readers and citable by the Digital Object Identifier (DOI®). "Just Accepted" is an optional service offered to authors. Therefore, the "Just Accepted" Web site may not include all articles that will be published in the journal. After a manuscript is technically edited and formatted, it will be removed from the "Just Accepted" Web site and published as an ASAP article. Note that technical editing may introduce minor changes to the manuscript text and/or graphics which could affect content, and all legal disclaimers and ethical guidelines that apply to the journal pertain. ACS cannot be held responsible for errors or consequences arising from the use of information contained in these "Just Accepted" manuscripts. 
Complexation Behavior of Polyelectrolytes and

Polyampholytes

\author{
Arun Kumar Narayanan Nair, Arturo Martinez Jimenez, and Shuyu Sun \\ Physical Science and Engineering Division (PSE), \\ Computational Transport Phenomena Laboratory, \\ King Abdullah University of Science and Technology (KAUST), \\ Thuwal, 23955-6900, Saudi Arabia.
} July 23,2017

\footnotetext{
*To whom correspondence should be addressed, email: arun.narayanannair@kaust.edu.sa
} 


\begin{abstract}
We perform grand canonical Monte Carlo simulations to study the $\mathrm{pH}$ titrations of isolated polyampholytes and polyelectrolyte-polyampholyte complexes in dilute solutions. Our simulations indicate that the electrostatic interactions promote the coexistence of opposite charges along the polyampholyte chain during titration. The repulsion between excess charges typically dominates the electrostatic interaction and leads to polymer stretching. Salt ions can screen the repulsion between excess charges as well as the fluctuation-induced attraction between opposite charges, and therefore make the variation between titration curves of polyampholytes and the ideal (no electrostatic interactions) curves less significant. We observe that this screening of charge repulsion decreases the chain size. The presence of pearl-necklace configuration of polyampholytes is diminished by the addition of salt. Similar simulations for the polyelectrolytepolyampholyte system show that the resulting complexes are generally stable in the low $\mathrm{pH}$ region. In comparison to ideal case, electrostatic interactions strongly influence the acid-base properties of polyampholyte chains in the adsorbed state by reducing the presence of the coexistence domain of both positive and negative charges in the titration curves. We attribute the complex formation between polyelectrolyte and polyampholyte chains in the high $\mathrm{pH}$ region to, e.g., the high salt content. The $\mathrm{pH}$ variation leads to abrupt transition between adsorbed and desorbed states. Independent of charge sequence, a polyampholyte chain in a complex is usually located at one of the ends of the polyelectrolyte chain.
\end{abstract}




\section{Introduction}

Charged polymers containing ionizable groups can dissolve in polar solvents, leaving charged monomers on polymer backbone and releasing counterions into solution. They are classified into polyelectrolytes when the polymers carry either cationic or anionic monomers and as polyampholytes when the polymers are capable of carrying both positive and negative charges on the same chain. ${ }^{1-4}$ Many charged polymers found in nature, including proteins, are in fact polyampholytes. Depending on the different dissociation behavior one can distinguish between weak (also known as annealed) and strong (also known as quenched) polyelectrolytes. ${ }^{5}$ Weak polyelectrolytes such as poly(acrylic acid) dissociate in a limited pH range only. Typically a weak polyelectrolyte has slight charge depletion in the central part of the chain and the charges accumulate at the ends. ${ }^{6}$ Whereas strong polyelectrolytes such as poly(sodium styrene sulfonate), where the charge distribution along the backbone is solely imposed by polymer synthesis, dissociate completely in the total $\mathrm{pH}$ range accessible by experiment. The rich behavior of strong polyelectrolytes and polyampholytes is qualitatively different from that of uncharged polymers. ${ }^{2,3,7-15}$

Weak polyelectrolytes display a number of interesting and nontrivial conformational characteristics. ${ }^{16-20}$ Molecular simulations have shown that depending on the solvent quality for polymer backbone and $\mathrm{pH}$, polyelectrolytes exist in different conformations such as coil, globule, pearl-necklace, and stretched. Kirwan et al. ${ }^{21}$ gave the first direct experimental evidence that the variation of charge on weak polyelectrolytes can be a direct tool to induce pearl-necklaces between globular and extended structures. The pearl-necklace shape is similar to the instabilities exhibited by a charged droplet. ${ }^{22}$ For weak polyelectrolytes in a poor solvent, a first-order conformational transition from a globule to an extended state of the chain predicted by theory ${ }^{5}$ was confirmed by simulations ${ }^{18,20}$ and experiments. ${ }^{23}$

Similar to the behavior exhibited by polyelectrolytes, polyampholytes also display intriguing conformational features. In the $\mathrm{pH}$-solvent quality plane, polymers 
showed extended as well as globular conformations, and weakly charged block has coiled or extended structures, whereas the strongly charged block has extended conformations. ${ }^{20}$ The presence of one oppositely charged nanoparticle significantly modified the acid-base properties of the weak polyelectrolyte. ${ }^{16}$ A similar situation was obtained for polyampholytes, ${ }^{20}$ where the presence of an oppositely charged block significantly modified the acid (base) property of the other block by promoting the formation of negatively (positively) charged monomers over the whole $\mathrm{pH}$ range. A discontinuous transition between extended and collapsed conformations of polyampholytes varying the $\mathrm{pH}$ of solution can be obtained due to the first-order-like nature of the transition.

The adsorption of polyelectrolytes and polyampholytes on to charged chains and surfaces has been under extensive theoretical study for past few years. ${ }^{2,3,11,12}$ The considerable interest in this problem stems from its importance in biology, materials science, soft matter research, and oil and gas industry. Complexes between polyelectrolytes or nanoparticles and polyampholytes play a significant role in protein purification and separation, ${ }^{24,25}$ DNA-protein interaction, ${ }^{26,27}$ lubrication by synovial fluid, ${ }^{28}$ biosensor development, ${ }^{29}$ enzyme immobilization, ${ }^{30}$ and oil production. ${ }^{31,32}$ Polymers showed characteristic adsorption properties because of their flexibility and internal degrees of freedom, when attracted to surfaces. ${ }^{11}$ The different surface geometries yielded very distinct scaling laws for the critical surface charge density required to initiate chain adsorption. ${ }^{12}$ Adsorption processes involving charged chains and nanoparticles or surfaces are commonly studied by computer simulations. ${ }^{13,14,16,33,34}$ A remarkably good agreement between theory and simulation was obtained for the adsorption behavior of polyelectrolytes on to spherical surfaces. ${ }^{12,34}$ When the macroion-polyelectrolyte interactions were not enough to overcome the entropic penalty of polymer confinement, the conformational properties of the chain showed abrupt variations as a result of first-order-like transition between the adsorbed and desorbed states. ${ }^{33}$

Recently molecular simulations were carried out to investigate the complexation behavior of polyampholytes with negatively charged nanoparticles. ${ }^{15,35,36}$ The adsorption of the polyampholyte chain at the nanoparticle surface was difficult to achieve, 
e.g., at low surface charge densities of the nanoparticle. By increasing the charge density and the nanoparticle size, the polyampholyte chain collapsed on to the surface of the nanoparticle. ${ }^{15}$ Increasing the size of the charged blocks in the polyampholyte chain resulted in a decrease of the $\mathrm{pH}_{\text {critic }}$ value ( $\mathrm{pH}$ value at which desorption is observed when $\left.\mathrm{pH} \leq \mathrm{pH}_{\text {critic }}\right)$ and thus increases the complex formation domain. ${ }^{35}$ In the case of strongly hydrophobic chains, no adsorption with the nanoparticle was observed within the relevant $\mathrm{pH}$ range. The adsorption process occurred with chains that are electrostatically more charged and less hydrophobic. ${ }^{36}$

The polymer and salt concentrations, the charge distribution along the polyampholyte backbone, and the solvent quality for the polymer backbone play an important role in determining the structure of a polyelectrolyte-polyampholyte complex (e.g., DNA-protein complex). ${ }^{9,10}$ A polyampholyte chain in a complex is typically located at one of the ends of the polyelectrolyte and aligned along the polyelectrolyte backbone at infinite dilution. ${ }^{9}$ Upon forming a complex with polyampholytes, a polyelectrolyte chain changes its necklace conformation by forming one huge bead in poor solvent. ${ }^{10}$ However, little is known about the complex formation between polyelectrolyte and polyampholyte chains as a function of the varying solution $\mathrm{pH} .{ }^{37-39}$

Our studies showed that molecular simulations constitute a powerful tool to generally explore the chemical and surface interactions. ${ }^{7,8,13,14,20,40}$ In this paper, we study the titration behavior and conformational properties of isolated polyampholytes and polyelectrolyte-polyampholyte complexes in dilute solutions. Simulations typically involve detailed molecular models of solvent, proteins, and DNA, and study dynamics of the system on the ps or ns time scale or on the level of coarse-grained models that significantly extends accessible time scales by sacrificing details of molecular structure. ${ }^{2,41}$ Within the framework of a coarse-grained model, ${ }^{2,20,42-44}$ we employ grand canonical Monte Carlo (GCMC) simulations to investigate the titration behavior of these chains, where the charges are in contact with a reservoir of constant chemical potential given by the solution $\mathrm{pH}$. The present paper provides new insight into the $\mathrm{pH}$ titration curves, adsorption-desorption limit, and chain conformations of polyelectrolyte-polyampholyte 
system for different physicochemical conditions. Our results show that the electrostatic interactions promote the coexistence of both positive and negative charges along the isolated polyampholyte chain during titration, and that the resulting chain size is in agreement with the scaling predictions. The adsorption of a polyelectrolyte chain onto the polyampholyte chain reduces the presence of this coexistence domain in the titration curves. Polyelectrolyte-polyampholyte complexes are not only stable in the low $\mathrm{pH}$ region but also in the high $\mathrm{pH}$ region, e.g., due to the presence of salt. We also find that the polyampholyte in a complex, independent of its charge sequence, is typically located at one of the ends of the polyelectrolyte chain. The outline of the paper is as follows: In Sec. II, we describe the model of our flexible chains and the details of the Monte Carlo simulations. The simulation results are presented in Sec. III and a brief summary as well as our conclusions can be found in Sec. IV.

\section{Simulation details}

We performed molecular simulations of complexation between polyelectrolyte (PE) and polyampholyte (PA) chains. Since the simulation model and method are the same as those used in our previous study, ${ }^{20}$ we briefly describe them here. The polymer chains are modeled as bead-spring chains of charged Lennard-Jones (LJ) particles with diameter $\sigma .{ }^{20,42}$ Polyelectrolyte in a complex has $N_{\mathrm{PE}}=128$ beads, all of which remain negatively charged during the whole simulation. For all investigated systems, polyampholyte chains in a complex contain $N_{\mathrm{PA}}=128$ repeat units and two different type of monomers (A and $\mathrm{B}$ ). A monomer of type $\mathrm{A}$ is considered to be an acid (each monomer carries zero charge in the protonated state and one negative unit of charge in the deprotonated state) and a monomer of type B represents a base (each monomer carries zero charge in the deprotonated state and one positive unit of charge in the protonated state). In this study, the number of monomers of type $\mathrm{A}$ is equal to the number of monomers of type B. The acid and base units are distributed in a random or blocky (diblock) fashion along the polyampholyte backbone. For comparison purposes, 
separate simulations were performed using isolated polyampholyte chains consisting of $N_{\mathrm{PA}}=256$ monomers.

All monomers interact via a truncated-shifted LJ potential

$$
U_{\mathrm{LJ}}\left(r_{i j}\right)= \begin{cases}4 \epsilon_{\mathrm{LJ}}\left[\left(\frac{\sigma}{r_{i j}}\right)^{12}-\left(\frac{\sigma}{r_{i j}}\right)^{6}-\left(\frac{\sigma}{R_{\mathrm{C}}}\right)^{12}+\left(\frac{\sigma}{R_{\mathrm{C}}}\right)^{6}\right] ; & r_{i j} \leq R_{\mathrm{C}} \\ 0 ; & r_{i j}>R_{\mathrm{C}}\end{cases}
$$

where $r_{i j}$ is the distance between the centers of $i$ and $j$ particles and $R_{\mathrm{C}}$ is the cutoff radius. The parameter $\epsilon_{\mathrm{LJ}}$ controls the strength of the short-range interactions, and the LJ diameter $\sigma$ is used to set the length scale. The cutoff distance $R_{\mathrm{C}}=2.5 \sigma$ in all studied systems and the $\Theta$ point corresponds to $\epsilon_{\mathrm{LJ}}^{\Theta} \approx 0.34 k_{\mathrm{B}} T$ for the uncharged system. ${ }^{42}$ The interaction parameters for LJ interactions are summarized in Table 1. The choice of the LJ parameter $\epsilon_{\mathrm{LJ}}$ higher than $0.34 k_{\mathrm{B}} T$ for the polymer chains corresponds to poor solvent conditions for the polymer backbone. Our LJ parameters are consistent with those reported for the study of quenched polyampholyte chains in the presence of polyelectrolyte chains. ${ }^{10}$ Note that the solvent conditions can significantly affect the equilibrium conformations of polyelectrolyte-polyampholyte complexes. ${ }^{9,10}$

The connectivity of the bonded monomers is maintained by the finite extensible nonlinear elastic (FENE) potential

$$
U_{\mathrm{FENE}}\left(r_{i j}\right)=-\frac{1}{2} k_{\mathrm{FENE}} R_{0}^{2} \ln \left(1-\frac{r_{i j}^{2}}{R_{0}^{2}}\right)
$$

where the spring constant $k_{\mathrm{FENE}}=7 k_{\mathrm{B}} T / \sigma^{2}$ and the maximum bond length $R_{0}=$ $2 \sigma .{ }^{42,43}$ This potential provided rather stiff bond lengths, which made bond crossing energetically infeasible. ${ }^{42}$ The solvent is modeled as a continuous dielectric medium with dielectric constant $\epsilon$. The charged monomers are thus interacting with each other via the Debye-Hückel (DH) potential

$$
U_{\mathrm{DH}}\left(r_{i j}\right)=k_{\mathrm{B}} T \frac{\lambda_{\mathrm{B}} q_{i} q_{j}}{r_{i j}} \exp \left(-r_{i j} / \lambda_{\mathrm{D}}\right),
$$

where $q_{i}\left(q_{j}\right)$ is the charge valence of the $i$ th $(j$ th $)$ particle, equal to $0,-1$, and +1 for the neutral, negatively charged, and positively charged monomers, respectively. The Bjerrum length $\lambda_{\mathrm{B}}=e^{2} /\left(\epsilon k_{\mathrm{B}} T\right)$, where $e$ is the elementary charge, determines the strength 
of the electrostatic interactions and the Debye screening length $\lambda_{\mathrm{D}}=\left(8 \pi \mathrm{c}_{\mathrm{s}} \lambda_{\mathrm{B}}\right)^{-1 / 2}$, where $c_{\mathrm{s}}$ is the salt concentration (1:1 electrolyte). The value of the Bjerrum length has to be chosen as $\lambda_{\mathrm{B}} \lesssim \sigma$ to avoid problems with counterion condensation due to the fact that counterions are not treated explicitly in our model. ${ }^{43}$ The length scale of our coarse-grained model is set by the Bjerrum length $\lambda_{\mathrm{B}}$, which is about $0.7 \mathrm{~nm}$ for water at room temperature. In our simulation, the average bond length is given by $b \approx \sigma$. The average distance between neighboring charges along the backbone is about $0.7 \mathrm{~nm}$ for the fully ionized chain at $\lambda_{\mathrm{B}}=\sigma$. Therefore, the contour length of the chain is about $0.2 \mu \mathrm{m}$ (256 monomers) in our simulation, while that of, e.g., a fully stretched $190 \mathrm{kDa}$ poly(vinylamine) chain is about $1 \mu \mathrm{m} .{ }^{21}$ Nevertheless, our choice of the number of monomers in a chain is consistent with previous simulation studies of charged polymers in dilute solutions. ${ }^{16,18,19,35,36}$

The annealed polyampholyte is simulated in a grand canonical ensemble where the chain is in contact with a reservoir of charges of fixed chemical potential imposed by the solution $\mathrm{pH}$ via ${ }^{35,45}$

$$
\frac{\mu}{k_{\mathrm{B}} T}=\left\{\begin{array}{lc}
-\ln 10\left(\mathrm{pH}-\mathrm{pK}_{0}^{\mathrm{A}}\right)-\left(\lambda_{\mathrm{B}} / \lambda_{\mathrm{D}}\right) ; & (\text { acid }) \\
\ln 10\left(\mathrm{pH}-\mathrm{pK}_{0}^{\mathrm{B}}\right)-\left(\lambda_{\mathrm{B}} / \lambda_{\mathrm{D}}\right) ; & (\text { base }) .
\end{array}\right.
$$

Our model has no direct connection to any specific polyampholyte and the values of $\mathrm{pK}_{0}^{\mathrm{A}}$ and $\mathrm{pK}_{0}^{\mathrm{B}}$ are set to 5.5 and 7.5, respectively. Equilibrium properties are studied by using standard Metropolis Monte Carlo (MC) simulation. We employ different configurational MC moves such as local and pivot moves. During local moves a random displacement is given to a randomly picked monomer. In a pivot move, the randomly selected chain section on either side of a randomly selected monomer is randomly rotated around that monomer. In addition, the algorithm is completed with a charge move by which the charge state of a randomly picked monomer is switched. The energy change of a complete MC move reads

$$
\Delta E=\Delta U_{\mathrm{LJ}}+\Delta U_{\mathrm{FENE}}+\Delta U_{\mathrm{DH}} \pm \mu
$$

The \pm sign in eq. (5) was changed depending on whether a protonation or deprotona- 
tion was attempted.

To ensure that the simulations generate equilibrium data we start from both straight and random initial conformations and require that after appropriate relaxation they yield identical averaged quantities. Typically, a polyampholyte chain in a complex is initially placed in the middle or near the ends of a polyelectrolyte chain. The final results are independent of the initial location of the polyampholyte and polyelectrolyte chains. Each system is equilibrated for a period of $5 \times 10^{6}$ Monte Carlo steps per monomer $(\mathrm{MCM})$, and the physical properties of the chains are obtained by averaging over the production run of $3 \times 10^{6} \mathrm{MCM}$ after the equilibration. The results presented below are obtained by averaging the production data over at least ten simulation runs which started from different initial conformations. Finally, the relative error of the root mean square end-to-end distance was found to be typically less than $5 \%$ and the fraction of charges are reproducible within less than $2 \%$ independently of the conformation of the charged polymer.

\section{Results and discussion}

\subsection{Isolated polyampholytes}

GCMC simulations are carried out to understand the titration behavior and conformational properties of diblock and random polyampholytes. Simulated titration curves of these polyampholytes depicting the variation of the average charge fraction $f\left(f=\left(f_{\mathrm{A}}+f_{\mathrm{B}}\right) / 2\right.$, where $f_{\mathrm{A}}$ and $f_{\mathrm{B}}$ are the average charge fractions of monomers of type $\mathrm{A}$ and type $\mathrm{B}$, respectively) as a function of the solution $\mathrm{pH}$ are presented in Fig. 1 as symbols. The results are given for polyampholytes in near $\Theta$-solvent conditions for the polymer backbone (the LJ parameter $\epsilon_{\mathrm{LJ}}=0.5 k_{\mathrm{B}} T$ ). For brevity, only the results of monomers of type B are shown in Fig. 1 because titration curves of monomers of type $\mathrm{A}$ and type $\mathrm{B}$ are symmetric with respect to the isoelectric point $(\mathrm{pH}=6.5)$. Figs. $\mathrm{S} 1$ and $\mathrm{S} 2$ in the Supporting Information provide the corresponding 
titration data for other conditions (the LJ parameter: $\epsilon_{\mathrm{LJ}}=1.5 k_{\mathrm{B}} T$ and $\epsilon_{\mathrm{LJ}}=2.5 k_{\mathrm{B}} T$, respectively). As compared to the ideal case (the Bjerrum length $\lambda_{\mathrm{B}}=0$ ) represented by dashed lines in the above figures, electrostatic interactions strongly influence the acid-base properties of polyampholyte chains by promoting the coexistence domain of both positive and negative charges. A well pronounced plateau, where the $\mathrm{pH}$ remains almost constant in titration curves has often been related to a first-order transition between an elongated state and a compacted globule state. ${ }^{5,18}$ Analyzing our titration curves at the extreme $\mathrm{pH}$ values, it becomes evident that the variation of $f_{\mathrm{A}}$ or $f_{\mathrm{B}}$ becomes more sharp on increasing the value of the Bjerrum length $\lambda_{\mathrm{B}}$ (In experiments, instead the interaction parameter $u=\lambda_{\mathrm{B}} / b$ - the ratio of the Bjerrum length $\lambda_{\mathrm{B}}$ to the monomer size $b$, may be tuned by varying the distance between the ionizable groups).

To get an insight into the conformational changes of polyampholytes with $\mathrm{pH}$ (or with corresponding $f$ ), simulation results of the root-mean-square radius of gyration $R_{\mathrm{g}}$ as a function of $f$ are presented in Fig. 2. The results are given for polyampholytes in near $\Theta$-solvent conditions for the polymer backbone (the LJ parameter $\epsilon_{\mathrm{LJ}}=0.5 k_{\mathrm{B}} T$ ). Figs. S3 and S4 provide the corresponding chain sizes for other conditions (the LJ parameter: $\epsilon_{\mathrm{LJ}}=1.5 k_{\mathrm{B}} T$ and $\epsilon_{\mathrm{LJ}}=2.5 k_{\mathrm{B}} T$, respectively). Furthermore, typical simulation snapshots that illustrate equilibrium chain structures in the $\mathrm{pH}$-solvent quality for the polymer backbone plane are shown in Fig. 3. Our simulation results indicate the presence of both collapsed and extended chain conformations. The globular conformation of diblock and random polyampholytes dominates the phase diagram over the whole pH range, especially for large values of the Lennard-Jones interaction parameter $\left(\epsilon_{\mathrm{LJ}}>0.34 k_{\mathrm{B}} T\right)$. Note that the fluctuation-induced electrostatic attraction is the driving force for the collapse of a polyampholyte chain under $\Theta$-solvent condition. ${ }^{2,20,44}$ These above observations are in line with our finding ${ }^{20}$ of the first-order like transition of the polyampholytes between the extended and the compact states, in the poor solvent conditions for the polymer backbone and at the extreme $\mathrm{pH}$ values.

As shown in Fig. 2a, the size of each block of the diblock polyampholyte has a nonmonotonic variation with the average charge fraction of the same block in the near 
$\Theta$-solvent conditions for the polymer backbone and for relatively high values of the Bjerrum length $\lambda_{\mathrm{B}}$. This result is qualitatively consistent with previous finding ${ }^{20}$ in the good solvent conditions for the polymer backbone. This behavior is due to the fact that below the average charge fraction corresponding to the isoelectric point, each block follows either the single-chain polyelectrolyte-like behavior or the polyelectrolyte-like behavior due to repulsion between uncompensated charges $\left(N_{\mathrm{PA}} \delta f=N_{\mathrm{PA}}\left|f_{\mathrm{A}}-f_{\mathrm{B}}\right|\right)$ along the chain. In the following discussion, we present scaling analysis neglecting all numerical coefficients and provide a comparison to our data. For example, for a polyampholyte chain of $N_{\mathrm{PA}}$ monomers, electrostatic interaction dominates on length scales larger than the electrostatic blob size $\xi_{\mathrm{e}}{ }^{2,3}$ Unperturbed by the electrostatic interactions, the chain statistics at the length scales smaller than the electrostatic blob size is Gaussian. Therefore, the electrostatic blob size $\xi_{\mathrm{e}}$ and the corresponding number of monomers $g_{\mathrm{e}}$ in it are determined by the balance between the electrostatic energy, $k_{\mathrm{B}} T \lambda_{\mathrm{B}}\left(\delta f g_{\mathrm{e}}\right)^{2} \xi_{\mathrm{e}}^{-1}$ and the thermal energy, $k_{\mathrm{B}} T$, where $\xi_{\mathrm{e}}$ follows the scaling law of a neutral chain, $\xi_{\mathrm{e}} \approx b g_{\mathrm{e}}^{1 / 2}$. This leads to the well-known scaling relations, $\xi_{\mathrm{e}} \approx b\left(u \delta f^{2}\right)^{-1 / 3}$ and $g_{\mathrm{e}} \approx\left(u \delta f^{2}\right)^{-2 / 3}$. The chain can be envisioned as an array of the electrostatic blobs which repel each other to form a fully extended chain of size $R_{\mathrm{g}} \approx\left(N_{\mathrm{PA}} g_{\mathrm{e}}^{-1}\right) \xi_{\mathrm{e}} \approx N_{\mathrm{PA}} b\left(u \delta f^{2}\right)^{1 / 3}$. As expected, such a behavior of polyampholytes becomes less pronounced on decreasing the value of the Bjerrum length $\lambda_{\mathrm{B}}$. At similar conditions, the chain size of random polyampholytes decreases with increasing $f_{\mathrm{A}}$ or $f_{\mathrm{B}}$ below the average charge fraction corresponding to the isoelectric point (see Fig. 2b). Here also the repulsion between excess charges dominates the electrostatic interaction and cause polymer stretching when the energy of this repulsion exceeds $k_{\mathrm{B}} T$. The insets in Fig. 2 summarizes the above three characteristic cases. Note that the size of a collapsed polyampholyte chain increases with decreasing electrostatic interactions due to weakening of fluctuation-induced attractions between charged monomers. For example, polyampholyte chains show Gaussian statistics at $\xi_{\mathrm{e}}>b N_{\mathrm{PA}}^{1 / 2}$, where the electrostatic blob size $\xi_{\mathrm{e}} \approx b\left(u f^{2}\right)^{-1 / 3}$ (diblock) $/ \xi_{\mathrm{e}} \approx b(u f)^{-1}$ (random). ${ }^{2,5,20,44}$ The above condition can be written in terms of the interaction parameter as $u<N_{\mathrm{PA}}^{-3 / 2} f^{-2}$ 
(diblock) $/ u<N_{\mathrm{PA}}^{-1 / 2} f^{-1}$ (random).

Interestingly, stable pearl-necklace structures are present in the phase diagram (see Fig. 3). The pearl-necklace structure of weak polyelectrolytes is stable if the solvent is not too poor $\left(\tau<\left(u^{3} N_{\mathrm{PE}}\right)^{-1 / 5}\right.$, where the solvent quality is measured by the dimensionless distance from the $\Theta$-point, $\tau=1-T / \Theta) .{ }^{5,17,20}$ In a poorer solvent $\left(\tau>\left(u^{3} N_{\mathrm{PE}}\right)^{-1 / 5}\right)$, the chemical potential is a nonmonotonic function of $f$, and the system exhibits a discontinuous transition between an elongated state and a compacted globule state. Polyampholytes form a pearl-necklace structure when the repulsion between excess charges $F_{\mathrm{el}} \approx N_{\mathrm{PA}}^{2} \delta f^{2} u R_{\mathrm{g}}^{-1}$ becomes comparable with the globular surface energy $F_{\text {surf }} \approx R_{\mathrm{g}}^{2} \xi_{\mathrm{e}}^{-2}\left(\Theta\right.$-solvent) $/ F_{\text {surf }} \approx R_{\mathrm{g}}^{2} \xi_{\mathrm{t}}^{-2}$ (poor solvent), where the thermal blob size $\xi_{\mathrm{t}} \approx b \tau^{-1} \cdot{ }^{2,5,20,44}$ In $\Theta$-solvent, collapsed polyampholytes with $\delta f<f^{1 / 3} N_{\mathrm{PA}}^{-1 / 2} u^{-1 / 3}$ (diblock) $/ \delta f<f^{1 / 2} N_{\mathrm{PA}}^{-1 / 2}$ (random) maintain a roughly spherical shape, while polyampholyte globules are unstable in the opposite limit. Excess charge deforms the polyampholyte globule into an elongated conformation at $\delta f>\tau^{1 / 2}\left(u N_{\mathrm{PA}}\right)^{-1 / 2}$ under poor solvent conditions. The pearl-necklace structure of polyampholytes is stable if the solvent is not too poor $\left(\tau<\left(u^{3} N_{\mathrm{PA}}\right)^{-1 / 5}\right)$, similar to the behavior of polyelectrolytes. In a poorer solvent $\left(\tau>\left(u^{3} N_{\mathrm{PA}}\right)^{-1 / 5}\right)$ polyampholytes exhibit a discontinuous transition between an elongated state and a compacted globule state. ${ }^{20}$ In agreement with these scaling predictions, the simulation snapshots show that the pearl-necklace morphology in the near $\Theta$-solvent conditions for the polymer backbone is stable. We clearly observe that the excess charges deform the collapsed polyampholyte chains into such a structure as $\mathrm{pH}$ is varied. In a poorer solvent, the globular conformation of diblock and random polyampholytes dominates the phase diagram. Though not shown in Fig. 3, we see that generally pearl-necklace structure is stable as the value of the Bjerrum length $\lambda_{\mathrm{B}}$ decreases.

Using the proton bath in the experiments may introduce electrostatic screening for polyions and therefore such models may not be treated as salt-free solutions. In this study, the influence of the ionic concentration is described through the Debye screening length $\lambda_{\mathrm{D}}$. Titration curves of polyampholytes in the presence of salt $\left(\lambda_{\mathrm{D}}=\sigma\right)$ are 
shown in Fig. 4, and Figs. S5 and S6. An examination of the curves shows that the decay in the average charge fractions of monomers of type $\mathrm{A}$ and type $\mathrm{B}$ for all cases is faster than in the case where no salt is added. These results suggest that the addition of salt plays an important role in the dissociation behavior of the polyampholyte chains. Salt ions can screen the repulsion between excess charges as well as the fluctuation-induced attraction between opposite charges, ${ }^{2}$ and therefore make the difference between titration curves of polyampholytes and the ideal curves less important.

Simulation results of chain sizes and snapshots that illustrate the equilibrium chain structures in the presence of salt are presented in Figs. 5 (the LJ parameter $\left.\epsilon_{\mathrm{LJ}}=0.5 k_{\mathrm{B}} T\right)$ and 6 , respectively. Figs. S7 and S8 provide the corresponding chain sizes for other conditions (the LJ parameter: $\epsilon_{\mathrm{LJ}}=1.5 k_{\mathrm{B}} T$ and $\epsilon_{\mathrm{LJ}}=2.5 k_{\mathrm{B}} T$, respectively). The repulsion between uncompensated charges of a polyampholyte chain begins to be screened once the Debye length $\lambda_{\mathrm{D}}$ becomes smaller than the size of the chain. We find that this screening of charge repulsion decreases the chain size, with all other parameters fixed (compare, e.g., Figs. 2 and 5). Due to the polyelectrolytelike behavior, the size of the polyampholyte chain $R_{\mathrm{g}} \approx b\left(u \delta f^{2}\right)^{1 / 5} N_{\mathrm{PA}}^{3 / 5}\left(\lambda_{\mathrm{D}} b^{-1}\right)^{2 / 5}$ $\left(\xi_{\mathrm{e}}<\lambda_{\mathrm{D}}<R_{\mathrm{g}}\right) .{ }^{46}$ An additional simulation of a quenched polyelectrolyte chain with $f=1$ confirmed the scaling relation $R_{\mathrm{g}} \sim \lambda_{\mathrm{D}}^{2 / 5}$ for our polymer model (data not shown). The size of a collapsed polyampholyte chain increases with increasing salt concentration $\left(b<\lambda_{\mathrm{D}}<\xi_{\mathrm{e}}\right)$ near $\Theta$-solvent conditions for the polymer backbone. ${ }^{2}$ This is because the fluctuation-induced attraction between charged monomers is screened by addition of salt. As in the case of polyelectrolytes, ${ }^{19}$ the cylindrical or pearl-necklace configuration of polyampholytes is reduced to the region $b^{3 / 2} u^{-1 / 2} \lambda_{\mathrm{D}}^{-3 / 2}<\delta f<\tau^{3 / 2} u^{-1 / 2}$ for values of the Debye length $\lambda_{\mathrm{D}}$ smaller than the globule size $R_{\mathrm{g}} \approx b N_{\mathrm{PA}}^{1 / 3} \tau^{-1 / 3}$ but larger than the thermal blob size $\xi_{\mathrm{t}}$. In the $\mathrm{pH}$-solvent quality for the polymer backbone plane, collapsed conformation of polyampholytes remains stable despite the high charge asymmetry because electrostatic interaction is weakened also by varying the value of the Bjerrum length $\lambda_{\mathrm{B}}$ (see Fig. 6). 


\subsection{Complexation behavior of polyelectrolytes and polyam- pholytes}

GCMC simulations are performed to understand the titration behavior of diblock and random polyampholytes in the presence of a negatively charged polyelectrolyte. Simulated titration data of these polyampholytes are presented in Fig. 7 as symbols. Titration curves are shown for monomers of type A and type B, and corresponding ideal curves (dotted and dashed lines, respectively) are also given for comparison. Figs. S9 and S10 provide the corresponding titration data in the presence of salt (the Debye length: $\lambda_{\mathrm{D}}=10 \sigma$ and $\lambda_{\mathrm{D}}=\sigma$, respectively). At relatively high values of the Bjerrum length $\lambda_{\mathrm{B}}$ and/or the Debye length $\lambda_{\mathrm{D}}$, we find that the presence of the negatively charged polyelectrolyte largely modifies the acid-base properties of the polyampholyte chains, resulting in asymmetric titration curves with regard to the isoelectric point. Similarly, titration curves of polyampholytes in the presence of a nanoparticle displayed an asymmetric shape which denotes a complex interplay of the inter- and intramolecular interactions. ${ }^{35}$ An interesting feature of the ionization degree behavior is the abrupt increase that occurs at $\mathrm{pH}$ values higher than the isoelectric point $(\mathrm{pH}=6.5)$. This variation will be further discussed below. Similar abrupt variation was observed in the case of the adsorption of weak polyelectrolyte chains onto oppositely charged spherical macroions. ${ }^{33}$ With decreasing values of the Bjerrum length $\lambda_{\mathrm{B}}$ and/or the Debye length $\lambda_{\mathrm{D}}$, smaller differences between the simulated and ideal curves are observed. This is because of the reduced electrostatic interaction between the charged monomers.

The approximate adsorption/desorption limit is obtained based on the probability distribution function of the distance between the center of masses of polyelectrolyte and polyampholyte chains $R_{\text {com }}$ (Fig. 8, and Figs. S11 and S12). We consider here the chains to be, e.g., adsorbed if they are always confined within the limit determined by the radius of gyration of chains. These results show that the maximum in the distribution function moves to larger distances as the value of the $\mathrm{pH}$ increases. Typically, polyelectrolyte-polyampholyte complexes are stable in the low $\mathrm{pH}$ region $(\mathrm{pH} \lesssim 6.5)$. 
A further increase of solution $\mathrm{pH}$ results in desorbed chains due to repulsive interaction between the polyampholyte and the negatively charged polyelectrolyte.

As compared to the ideal case, electrostatic interactions strongly influence the acid-base properties of polyampholyte chains (desorbed state) by promoting the coexistence domain of both positive and negative charges in the titration curves. The results from the titration behavior and conformational properties of isolated polyampholytes have been summarized in the previous section. As seen from, e.g., Fig. 7, in a polyelectrolyte-polyampholyte complex, electrostatic interactions influence the titration behavior of polyampholyte chains (adsorbed state) by demoting this coexistence region of opposite charges along the polyampholyte chain. That is, for polyampholytes in a complex, monomers of type B are mostly ionized, whereas neutralization of monomers of type A is significantly promoted here to reduce the repulsive interaction with the negatively charged polyelectrolyte. As a result, $f_{A}$ is found to be smaller than the ideal case at a given $\mathrm{pH}$ value. Recent studies have revealed that the $\mathrm{pH}$ variation in the low $\mathrm{pH}$ regime significantly modifies the subtle competition between repulsive monomer of type A-nanoparticle, attractive monomer of type B-nanoparticle and attractive monomer of type A-monomer of type B electrostatic interactions. ${ }^{36}$ Interestingly, we also find complex formation between polyelectrolyte and polyampholyte chains in the high $\mathrm{pH}$ region $(\mathrm{pH} \gtrsim 6.5)$ which is attributed to, e.g., the high salt content. Here, complexes formed between polyelectrolyte and diblock polyampholyte chains are stable over a wide $\mathrm{pH}$ range in comparison to the random case. Note that the complexation between polyampholyte and polyelectrolyte chains is generally driven by polarization-induced attractive interactions when the polyampholyte carry a net charge of about zero or the same sign as that of the polyelectrolyte. ${ }^{2,9}$

A direct way to determine the order of the phase transition is the histogram method of Lee and Kosterlitz. ${ }^{47}$ We obtained the probability distribution of the corresponding energy, $E=U_{\mathrm{LJ}}+U_{\mathrm{FENE}}+U_{\mathrm{DH}}+\mu($ acid $) N_{-}+\mu($ base $) N_{+},{ }^{45}$ where $N_{-}$and $N_{+}$are the number of charged monomers of type A and type B, respectively. The normalised distribution function $P(E)$ is related to the free energy via $\mathcal{A} / k_{\mathrm{B}} T=-\ln P(E)$. The free 
energy distributions have the double-minima structures as in our previous simulation studies, ${ }^{20}$ which may indicate that the polymers generally experience first-order-like transitions during the $\mathrm{pH}$ titrations (data not shown). Similarly, when the macroionpolyelectrolyte interactions were not enough to overcome the entropic penalty of polymer confinement, the conformational properties of the $\mathrm{pH}$-responsive polyelectrolyte chain showed abrupt variations as a result of first-order-like transition between the adsorbed and desorbed states. ${ }^{33}$

The simulation results of chain sizes and snapshots that illustrate the equilibrium chain structures are presented in Figs. 9 and 10, respectively. Figs. S13 and S14 provide the corresponding chain sizes in the presence of salt (the Debye length: $\lambda_{\mathrm{D}}=$ $10 \sigma$ and $\lambda_{\mathrm{D}}=\sigma$, respectively). In the low $\mathrm{pH}$ region, the sizes of polyelectrolytes and polyampholytes in a complex are largely unaffected by the changes in solution $\mathrm{pH}$ while keeping all other parameters fixed (see, e.g., Fig. 9). A polyelectrolyte chain in a complex is stretched out by electrostatic repulsion between similarly charged monomers for high values of the Bjerrum length $\lambda_{\mathrm{B}}$ and/or the Debye length $\lambda_{\mathrm{D}}$. However, here the polyelectrolyte size is smaller than that of the corresponding isolated polyelectrolyte chain because of the presence of polyampholytes. For a given $\mathrm{pH}$ value, the decrease in the values of the Bjerrum length $\lambda_{\mathrm{B}}$ and/or the Debye length $\lambda_{\mathrm{D}}$ leads to gradual contraction of the polyelectrolyte chain in a complex.

Polyampholyte chains in a complex are largely collapsed and unaffected by the changes in the values of the Bjerrum length $\lambda_{\mathrm{B}}$ and/or the Debye length $\lambda_{\mathrm{D}}$. Note, however, that the negatively charged block of the diblock polyampholyte in a complex is seen to be stretched out, consistent with earlier studies. ${ }^{2,9,10}$ Independent of charge sequence, a polyampholyte chain in a complex is usually located at one of the ends of the extended polyelectrolyte chain (see Fig. 10) in agreement with previous studies. ${ }^{9}$ The formation of such structures can result in maximization of the attractive and minimization of the repulsive electrostatic interactions. ${ }^{9,10}$ In dilute solutions, a chargesymmetric polyampholyte chain moved toward the middle of the polyelectrolyte as the polymer concentration increased. ${ }^{9}$ Further increase of the polymer concentration led 
to formation of multichain aggregates in solution. A major contribution to the binding free energy associated with polyelectrolyte-polyampholyte complexes is the increase in entropy due to counterion release. In our current simulations we consider complex formation at infinite dilution and neglect the effect of counterions. Previous studies have indicated that counterion release does not change the final complex structure in dilute solutions. ${ }^{9,10}$

The simulation employed here uses a screened Debye-Hückel interaction potential and therefore we avoid high charge densities where counterion condensation occurs. In order to study, e.g., the effects of counterion condensation and the correlation-induced attraction, explicit counterions were considered. ${ }^{10,48}$ The balance of the counterion osmotic pressure and the elastic restoring force of the end-grafted chains govern the thickness of the polyelectrolyte brush in the so-called osmotic regime. ${ }^{13,14}$ The structural properties of the starlike weak polyelectrolytes are predominantly determined by the osmotic pressure of counterions and the electrostatic interactions. ${ }^{49}$ We stress that our aim is to study qualitatively the interplay of hydrophobic/hydrophilic and electrostatic interactions in our model polymers as $\mathrm{pH}$ is varied. We plan to expand this study to incorporate the effect of explicit counterions on $\mathrm{pH}$-induced transition.

\section{Conclusions}

Using molecular simulations, we have studied the titration behavior and conformational properties of isolated polyampholytes and polyelectrolyte-polyampholyte complexes in dilute solutions. As compared to the ideal titration curves, electrostatic interactions promote the coexistence of both positive and negative charges along the polyampholyte chain. The size of each block of the diblock polyampholyte has a nonmonotonic variation with the average charge fraction of the same block in the near $\Theta$-solvent conditions for the polymer backbone. At similar conditions, the chain size of random polyampholytes decreases with increasing average charge fraction. The repulsion between excess charges generally dominates the electrostatic interaction and leads to polymer 
stretching. Such a behavior of polyampholytes becomes less pronounced on decreasing the value of the Bjerrum length $\lambda_{\mathrm{B}}$. Polyampholytes form a pearl-necklace structure when the repulsion between excess charges becomes comparable with the globular surface energy. The pearl-necklace structure of polyampholytes is stable if the solvent is not too poor. In a poorer solvent polyampholytes exhibit a discontinuous transition between an elongated state and a compacted globule state. ${ }^{20}$ Salt ions can screen the repulsion between excess charges as well as the fluctuation-induced attraction between opposite charges, and hence make the difference between titration curves of polyampholytes and the ideal curves less important. We find that this screening of charge repulsion decreases the chain size. The occurrence of pearl-necklace conformation of polyampholytes is reduced by the addition of salt.

Typically, polyelectrolyte-polyampholyte complexes are stable in the low pH region. As compared to the ideal case, electrostatic interactions strongly influence the acid-base properties of polyampholyte chains in the adsorbed state by demoting the coexistence domain of opposite charges in the titration curves. The complex formation between polyelectrolyte and polyampholyte chains in the high $\mathrm{pH}$ region is attributed to, e.g., the high salt content. The free energy distributions have the double-minima structures, which indicate that the polymers experience first-order-like transitions between adsorbed and desorbed states during the $\mathrm{pH}$ titrations. In the low $\mathrm{pH}$ region, the sizes of polyelectrolyte and polyampholyte chains in a complex are largely unaffected by the changes in solution $\mathrm{pH}$. A polyelectrolyte chain in a complex is stretched out by electrostatic repulsion between similarly charged monomers. The decrease in the values of the Bjerrum length $\lambda_{\mathrm{B}}$ and/or the Debye length $\lambda_{\mathrm{D}}$ leads to gradual contraction of the polyelectrolyte chain in a complex. Polyampholyte chains in a complex are mostly collapsed and unaffected by the changes in the values of the Bjerrum length $\lambda_{\mathrm{B}}$ and/or the Debye length $\lambda_{\mathrm{D}}$. However, the negatively charged block of the diblock polyampholyte in a complex is seen to be stretched out. Independent of charge sequence, a polyampholyte chain in a complex is usually located at one of the ends of the polyelectrolyte chain. 
1

2

3

4

5

6

7

8

9

10

11

12

13

14

15

16

17

18

19

20

21

22

23

24

25

26

27

28

29

30

31

32

33

34

35

36

37

38

39

40

41

42

43

44

45

46

47

48

49

50

51

52

53

54

55

56

57

58

59

60

\section{Acknowledgments}

The research reported in this publication was supported by funding from King Abdullah University of Science and Technology (KAUST), Kingdom of Saudi Arabia. A. M. J. and A. K. N. N. gratefully acknowledge computational facilities provided at KAUST. Supporting Information

Additional details of simulation analysis are provided in the Supporting Information. 


\section{References}

(1) Dautzenberg, H.; Jaeger, W.; Kötz, J.; Philipp, B.; Seidel, C.; Stscherbina, D. Polyelectrolytes: Formation, Characterization and Application; Hanser Publishers: Munich, Vienna, New York, 1994.

(2) Dobrynin, A. V.; Colby, R. H.; Rubinstein, M. Polyampholytes. J. Polym. Sci., Part B: Polym. Phys. 2004, 42, 3513-3538.

(3) Dobrynin, A. V.; Rubinstein, M. Theory of Polyelectrolytes in Solutions and at Surfaces. Prog. Polym. Sci. 2005, 30, 1049-1118.

(4) Kudaibergenov, S. E. Polyampholytes: Synthesis, Characterization, and Applications; Kluwer Academic/Plenum: New York, 2002, 186-197.

(5) Raphael, E.; Joanny, J.-F. Annealed and Quenched Polyelectrolytes. Europhys. Lett. 1990, 13, 623-628.

(6) Holm, C.; Joanny, J. F.; Kremer, K.; Netz, R. R.; Reineker, P.; Seidel, C.; Vilgis, T. A.; Winkler, R. G. Polyelectrolyte Theory. In Polyelectrolytes with Defined Molecular Architecture II; Springer Berlin Heidelberg, 2004, 67-111.

(7) Ganesan, V.; Kumar, N.A.; Pryamitsyn, V. Blockiness and Sequence Polydispersity Effects on the Phase Behavior and Interfacial Properties of Gradient Copolymers. Macromolecules 2012, 45 (15), 6281-6297.

(8) Stukalin, E.B.; Cai, L.H.; Kumar, N.A.; Leibler, L.; Rubinstein, M. Self-Healing of Unentangled Polymer Networks with Reversible Bonds. Macromolecules 2013, $46(18), 7525-7541$.

(9) Jeon, J.; Dobrynin, A.V. Molecular Dynamics Simulations of PolyampholytePolyelectrolyte Complexes in Solutions. Macromolecules 2005, 38 (12), 53005312 . 
(10) Jeon, J.; Dobrynin, A. V. Molecular Dynamics Simulations of PolyelectrolytePolyampholyte Complexes. Effect of Solvent Quality and Salt Concentration. J. Phys. Chem. B 2006, 110 (48), 24652-24665.

(11) Netz, R. R.; Andelman, D. Neutral and Charged Polymers at Interfaces. Phys. Rep. 2003, 380 (1), 1-95.

(12) Cherstvy, A. G.; Winkler, R. G. Polyelectrolyte Adsorption onto Oppositely Charged Interfaces: Unified Approach for Plane, Cylinder, and Sphere. Phys. Chem. Chem. Phys. 2011, 13 (24), 11686-11693.

(13) Ahrens, H., Frster, S., Helm, C.A., Kumar, N.A., Naji, A., Netz, R.R.; Seidel, C. Nonlinear Osmotic Brush Regime: Experiments, Simulations and Scaling Theory. J. Phys. Chem. B 2004, 108 (43), 16870-16876.

(14) Kumar, N.A.; Seidel, C. Polyelectrolyte Brushes with Added Salt. Macromolecules 2005, 38 (22), 9341-9350.

(15) Feng, J.; Ruckenstein, E. Monte Carlo Simulation of Polyampholyte-Nanoparticle Complexation. Polymer 2003, 44 (10), 3141-3150.

(16) Ulrich, S.; Laguecir, A.; Stoll, S. Complexation of a Weak Polyelectrolyte with a Charged Nanoparticle. Solution Properties and Polyelectrolyte Stiffness Influences. Macromolecules 2005, 38, 8939-8949.

(17) Castelnovo, M.; Sens, P.; Joanny, J.-F. Charge Distribution on Annealed Polyelectrolytes. Eur. Phys. J. E 2000, 1, 115-125.

(18) Uyaver, S.; Seidel, C. First-Order Conformational Transition of Annealed Polyelectrolytes in a Poor Solvent. Europhys. Lett. 2003, 64, 536-542.

(19) Uyaver, S.; Seidel, C. Effect of Varying Salt Concentration on the Behavior of Weak Polyelectrolytes in a Poor Solvent. Macromolecules 2009, 42, 1352. 
(20) Nair, A.K.N.; Uyaver, S.; Sun, S. Conformational Transitions of a Weak Polyampholyte. J. Chem. Phys. 2014, 141 (13), 134905.

(21) Kirwan, L. J.; Papastavrou, G.; Borkovec, M.; Behrens, S. H. Imaging the Coilto-Globule Conformational Transition of a Weak Polyelectrolyte by Tuning the Polyelectrolyte Charge Density. Nano Lett. 2004, 4 (1), 149-152.

(22) Rayleigh, L. XX. On the Equilibrium of Liquid Conducting Masses Charged with Electricity. Philos. Mag. 1882, 14, 184-186.

(23) Wang, S.; Zhao, J. First-Order Conformation Transition of Single Poly(2vinylpyridine) Molecules in Aqueous Solutions. J. Chem. Phys. 2007, 126, 091104.

(24) Dubin, P. L.; Gao, J.; Mattison, K. Protein Purification by Selective Phase Separation with Polyelectrolytes. Sep. Purif. Methods 1994, 23 (1), 1-16.

(25) Wang, Y. F.; Gao, J. Y.; Dubin, P. L. Protein Separation via Polyelectrolyte Coacervation: Selectivity and Efficiency. Biotechnol. Prog. 1996, 12 (3), 356-362.

(26) Mathews, C. K.; Van Holde, K. E. Biochemistry; Benjamin/Cummings Publishing: Redwood City, CA, 1990.

(27) Luger, K.; Maeder, A. W.; Richmond, R. K.; Sargent, D. F.; Richmond, T. J. Crystal Structure of the Nucleosome Core Particle at $2.8 \AA$ Resolution. Nature 1997, 389 (6648), 251-260.

(28) Swann, D. A.; Radin, E. L.; Nazimiec, M.; Weisser, P. A.; Curran, N.; Lewinnek, G. Role of Hyaluronic Acid in Joint Lubrication. Ann. Rheum. Dis. 1974, 33 (4), 318.

(29) Salloum, D. S.; Schlenoff, J. B. Protein Adsorption Modalities on Polyelectrolyte Multilayers. Biomacromolecules 2004, 5 (3), 1089-1096. 
(30) Xia, J.; Mattison, K.; Romano, V.; Dubin, P. L.; Muhoberac, B. B. Complexation of Trypsin and Alcohol Dehydrogenase with Poly(diallyldimethylammonium chloride). Biopolymers 1997, 41 (4), 359-365.

(31) Ezell, R. G.; McCormick, C. L. Electrolyte- and pH-Responsive Polyampholytes with Potential as Viscosity-Control Agents in Enhanced Petroleum Recovery. J. Appl. Polym. Sci. 2007, 104 (5), 2812-2821.

(32) Barati, R.; Johnson, S. J.; McCool, S.; Green, D. W.; Willhite, G. P.; Liang, J. T. Polyelectrolyte Complex Nanoparticles for Protection and Delayed Release of Enzymes in Alkaline pH and at Elevated Temperature during Hydraulic Fracturing of Oil Wells. J. Appl. Polym. Sci. 2012, 126 (2), 587-592.

(33) de Oliveira, V.M.; de Carvalho, S.J. Adsorption of pH-Responsive Polyelectrolyte Chains onto Spherical Macroions. Eur. Phys. J. E 2014, 37 (8), 1-7.

(34) Chodanowski, P.; Stoll, S. Polyelectrolyte Adsorption on Charged Particles: Ionic Concentration and Particle Size Effects - A Monte Carlo Approach. J. Chem. Phys. 2001, 115 (10), 4951-4960.

(35) Ulrich, S.; Seijo, M.; Carnal, F.; Stoll, S. Formation of Complexes between Nanoparticles and Weak Polyampholyte Chains. Monte Carlo Simulations. Macromolecules 2011, 44, 1661-1670.

(36) Carnal, F.; Clavier, A.; Stoll, S. Modelling the Interaction Processes between Nanoparticles and Biomacromolecules of Variable Hydrophobicity: Monte Carlo Simulations. Environ. Sci. Nano 2015, 2 (4), 327-339.

(37) Park, J.M.; Muhoberac, B.B.; Dubin, P.L.; Xia, J. Effects of Protein Charge Heterogeneity in Protein-Polyelectrolyte Complexation. Macromolecules 1992, 25 (1), 290-295.

(38) Mattison, K.W.; Dubin, P.L.; Brittain, I.J. Complex Formation between Bovine 
Serum Albumin and Strong Polyelectrolytes: Effect of Polymer Charge Density. J. Phys. Chem. B 1998, 102 (19), 3830-3836.

(39) Hattori, T.; Hallberg, R.; Dubin, P.L. Roles of Electrostatic Interaction and Polymer Structure in the Binding of -Lactoglobulin to Anionic Polyelectrolytes: Measurement of Binding Constants by Frontal Analysis Continuous Capillary Electrophoresis. Langmuir 2000, 16 (25), 9738-9743.

(40) Kadoura, A.; Nair, A. K. N.; Sun, S. Molecular Simulation Study of Montmorillonite in Contact with Variably Wet Supercritical Carbon Dioxide. J. Phys. Chem. C 2017, 121, 61996208.

(41) Giudice, E.; Lavery, R. Simulations of Nucleic Acids and Their Complexes. Acc. Chem. Res. 2002, 35 (6), 350-357.

(42) Micka, U.; Holm, C.; Kremer, K. Strongly Charged, Flexible Polyelectrolytes in Poor Solvents: Molecular Dynamics Simulations. Langmuir 1999, 15, 4033-4044.

(43) Limbach, H. J.; Holm, C. Single-Chain Properties of Polyelectrolytes in Poor Solvent. J. Phys. Chem. B 2003, 10\%, 8041-8055.

(44) Wang, Z.; Rubinstein, M. Regimes of Conformational Transitions of a Diblock Polyampholyte. Macromolecules 2006, 39, 5897-5912.

(45) Burak, Y.; Netz, R. R. Charge Regulation of Interacting Weak Polyelectrolytes. J. Phys. Chem. B 2004, 108, 4840-4849.

(46) Carrillo J. M.; Dobrynin, A. V. Polyelectrolytes in Salt Solutions: Molecular Dynamics Simulations. Macromolecules 2011, 44 (14), 5798-5816.

(47) Lee J.; Kosterlitz J. M. New Numerical Method To Study Phase Transitions. Phys. Rev. Lett. 1990, 65, 137.

(48) Panagiotopoulos, A. Z. Charge Correlation Effects on Ionization of Weak Polyelectrolytes. J. Phys.: Condens. Matter 2009, 21, 424113. 
1

2

3

4

5

6

7

8

9

10

11

12

13

14

15

16

17

18

19

20

21

22

23

24

25

26

27

28

29

30

31

32

33

34

35

36

37

38

39

40

41

42

43

44

45

46

47

48

49

50

51

52

53

54

55

56

57

58

59

60
(49) Uhlik, F.; Kosovan, P.; Limpouchova, Z.; Prochazka, K.; Borisov, O. V.; Leermakers, F. A. Modeling of Ionization and Conformations of Starlike Weak Polyelectrolytes. Macromolecules 2014, 47 (12), 40044016. 
Table 1: Interaction parameters used in simulations of polyelectrolyte-polyampholyte complexes.

Monomer pairs $\quad \epsilon_{\mathrm{LJ}} /\left(k_{\mathrm{B}} T\right) \quad R_{\mathrm{C}} / \sigma$

$\begin{array}{lll}\text { Polyelectrolyte-polyelectrolyte } & 1.5 & 2.5\end{array}$

$\begin{array}{lll}\text { Polyampholyte-polyampholyte } \quad 0.35 & 2.5\end{array}$

Polyelectrolyte-polyampholyte $\sqrt{(1.5 \times 0.35)} \quad 2.5$ 


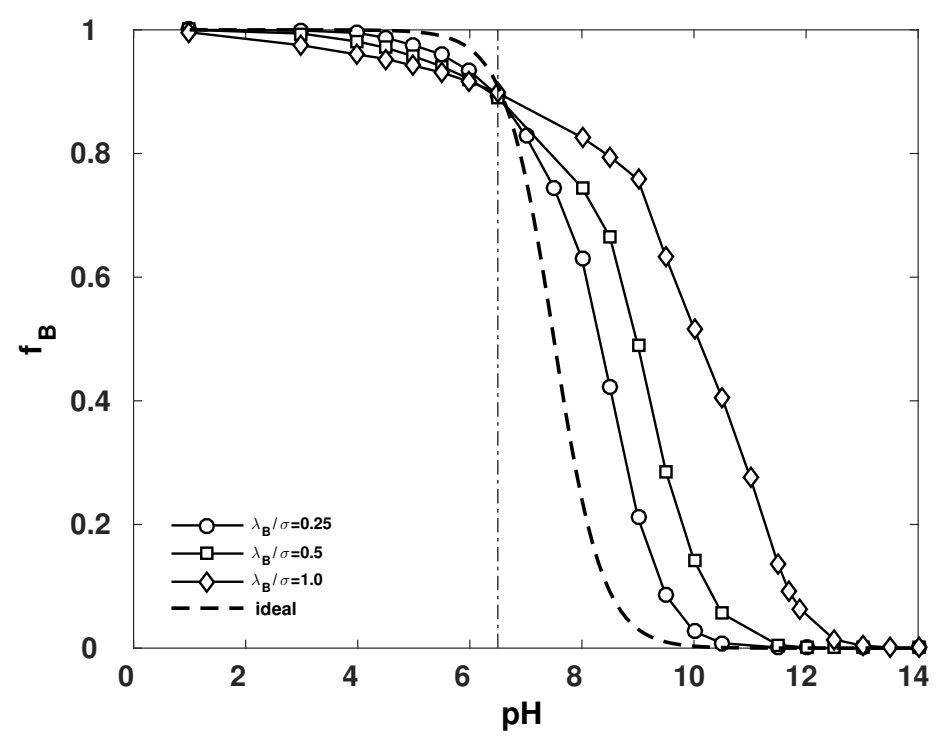

(a)

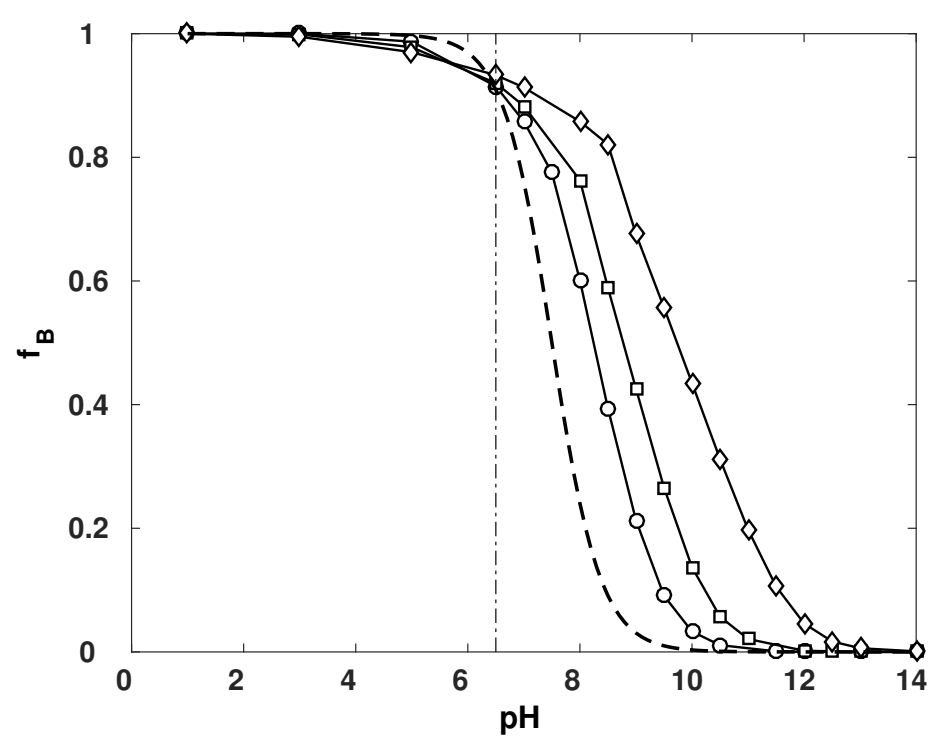

(b)

Figure 1: Titration curves of polyampholytes at different values of the Bjerrum length $\lambda_{\mathrm{B}}$. Shown is the average charge fraction of monomers of type $\mathrm{B} f_{\mathrm{B}}$ as a function of solution pH: (a) diblock polyampholyte and (b) random polyampholyte. The LJ parameter $\epsilon_{\mathrm{LJ}}=0.5 k_{\mathrm{B}} T$, and the values of $\mathrm{pK}_{0}^{\mathrm{A}}$ and $\mathrm{pK}_{0}^{\mathrm{B}}$ are 5.5 and 7.5 , respectively. 


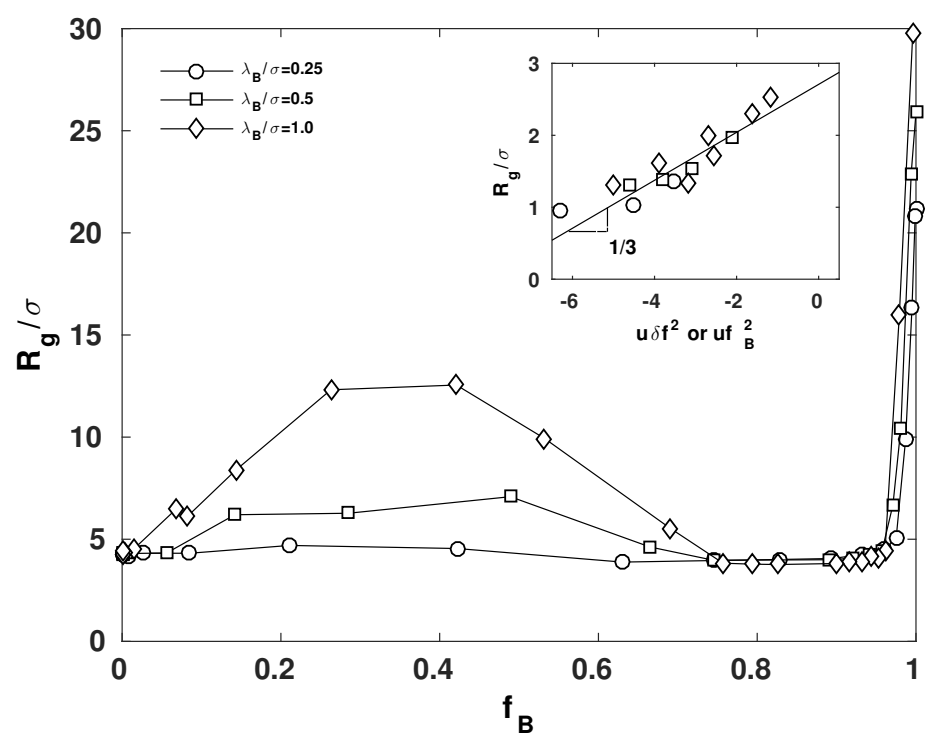

(a)

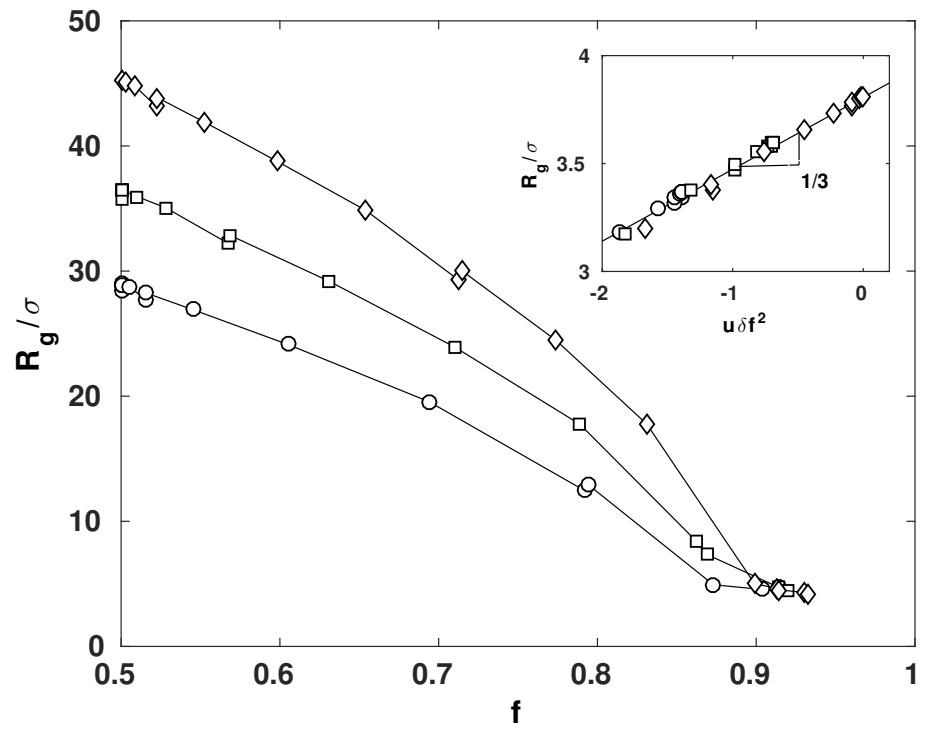

(b)

Figure 2: (a) Root-mean-square radius of gyration $R_{\mathrm{g}}$ of the block containing monomers of type $\mathrm{B}$ in the diblock polyampholyte as a function of the average charge fraction of monomers of type $\mathrm{B} f_{\mathrm{B}}$. (b) Root-mean-square radius of gyration $R_{\mathrm{g}}$ of the random polyampholyte as a function of the average charge fraction $f$. Symbols are the same as given in Fig. 1. Insets (log-log scale) show comparison of theory with the data in the main figures (see text). The data of $R_{\mathrm{g}}$ vs $u f_{\mathrm{B}}^{2}$ were shifted along the y-axis for clarity. 


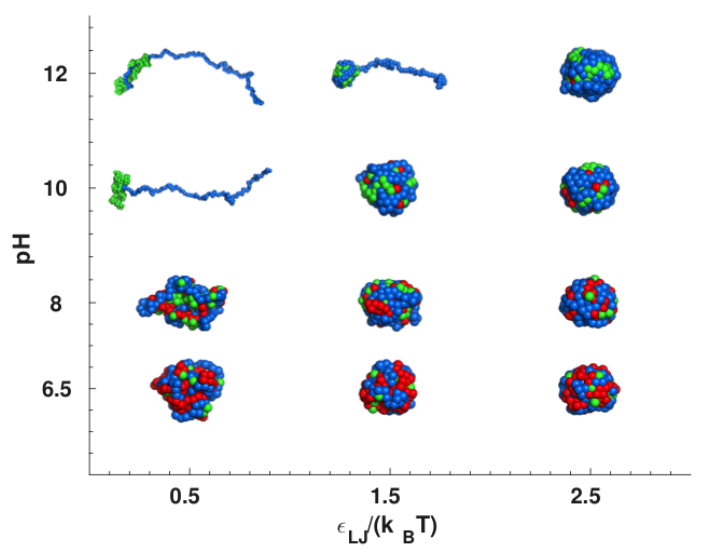

(a)

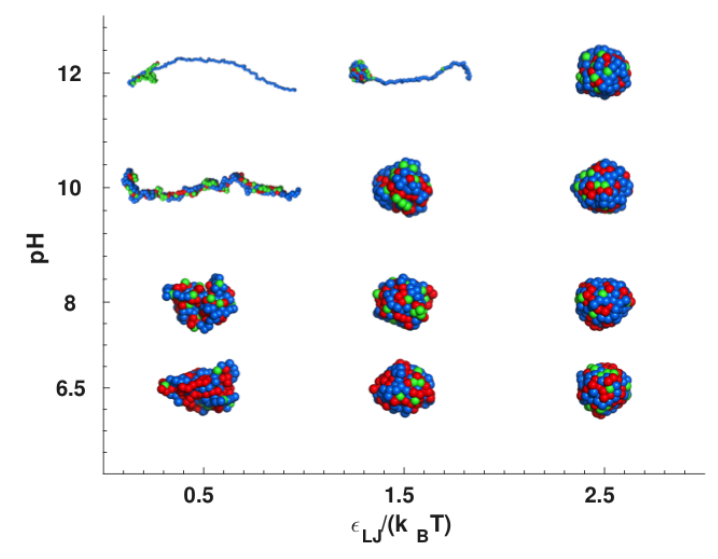

(b)

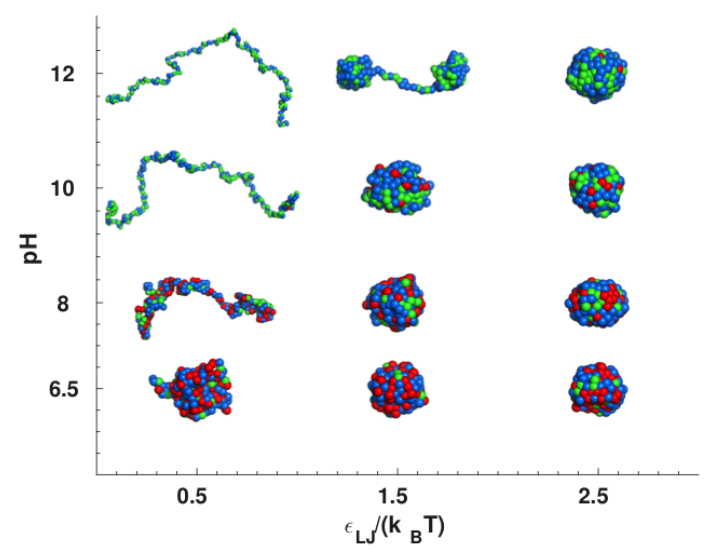

(c)

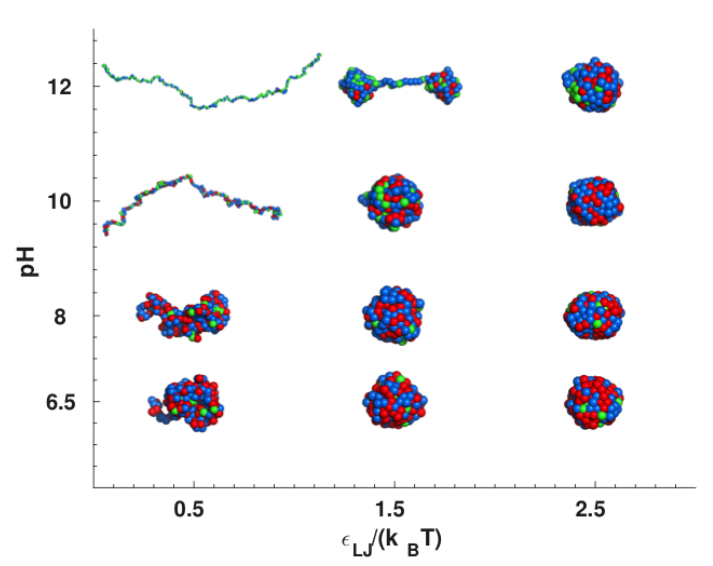

(d)

Figure 3: Typical conformations of diblock (left panels) and random (right panels) polyampholyte chains in the $\mathrm{pH}$-solvent quality (tuned by varying the LJ parameter $\epsilon_{\mathrm{LJ}}$ ) plane at the Bjerrum length $\lambda_{\mathrm{B}}=0.25 \sigma$ (top panels) and $\lambda_{\mathrm{B}}=\sigma$ (bottom panels). The neutral, negatively charged, and positively charged monomers are colored green, blue, and red, respectively. 


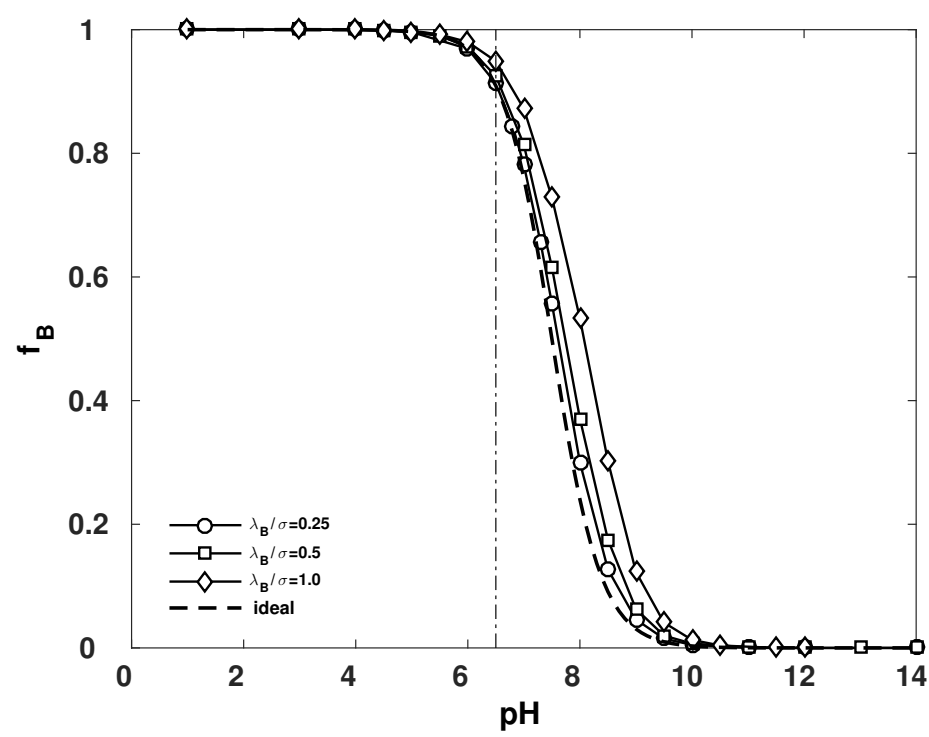

(a)

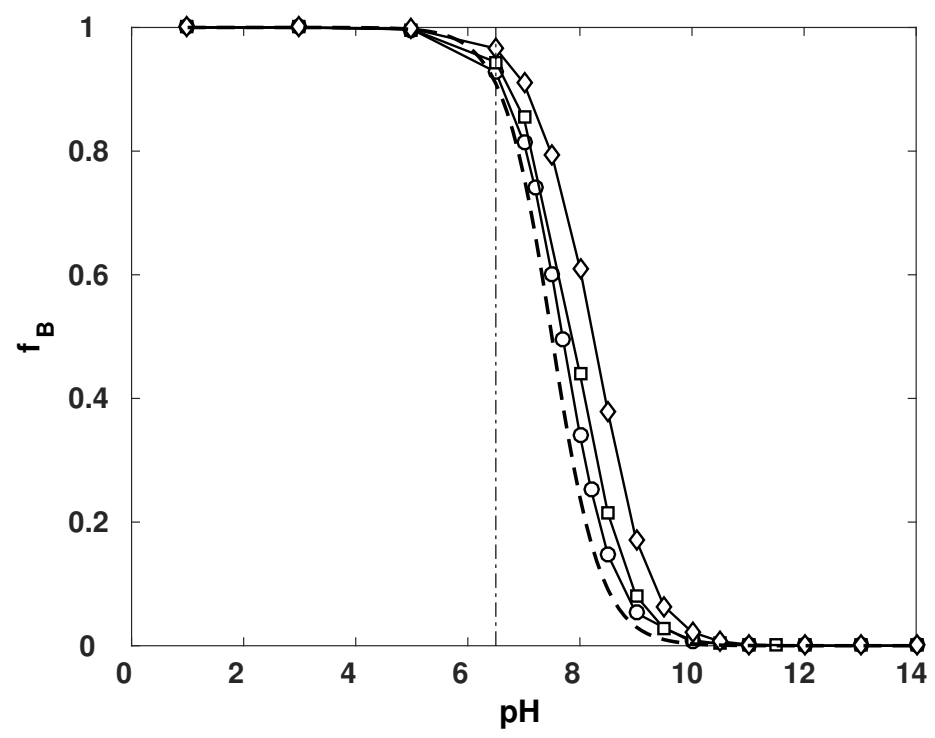

(b)

Figure 4: Same as in Fig. 1, but in the presence of salt (the Debye length $\lambda_{\mathrm{D}}=\sigma$ ). 


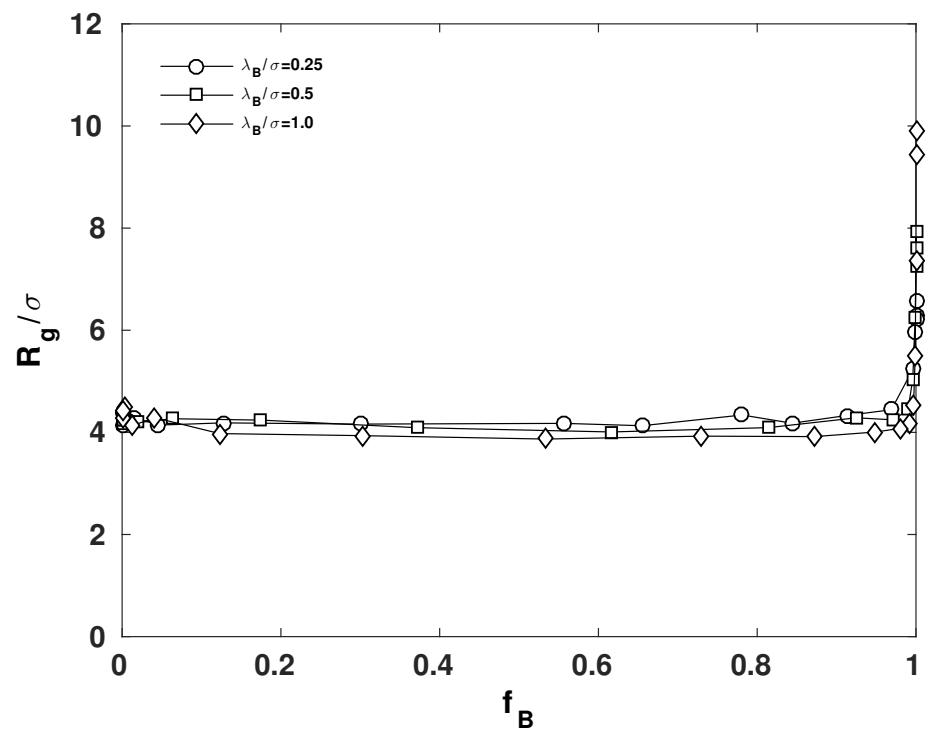

(a)

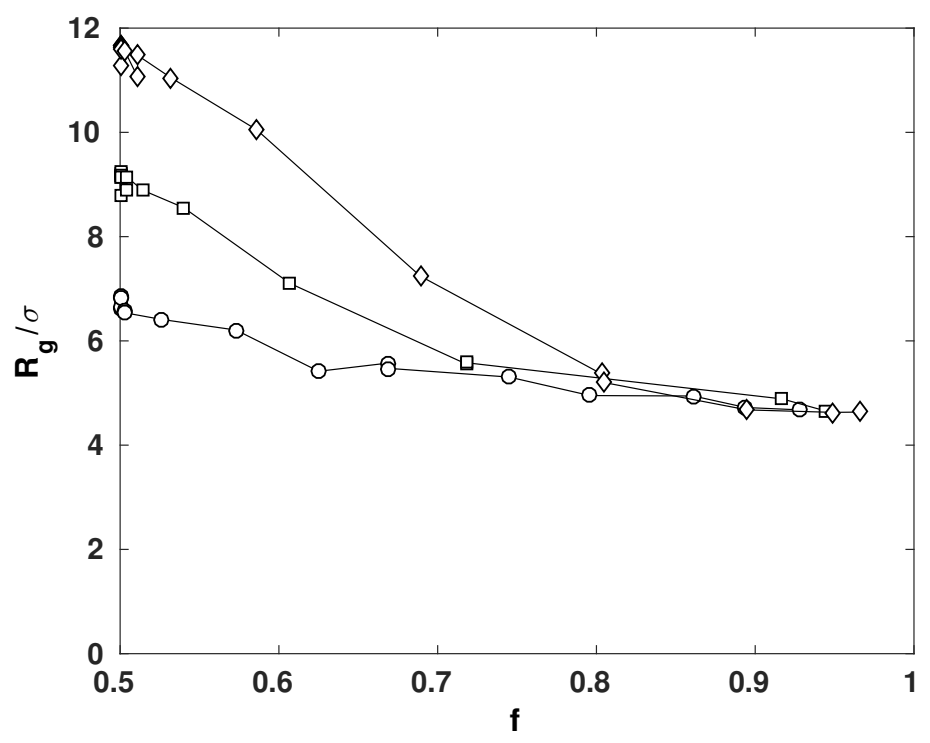

(b)

Figure 5: Same as in Fig. 2, but in the presence of salt (the Debye length $\lambda_{\mathrm{D}}=\sigma$ ). 


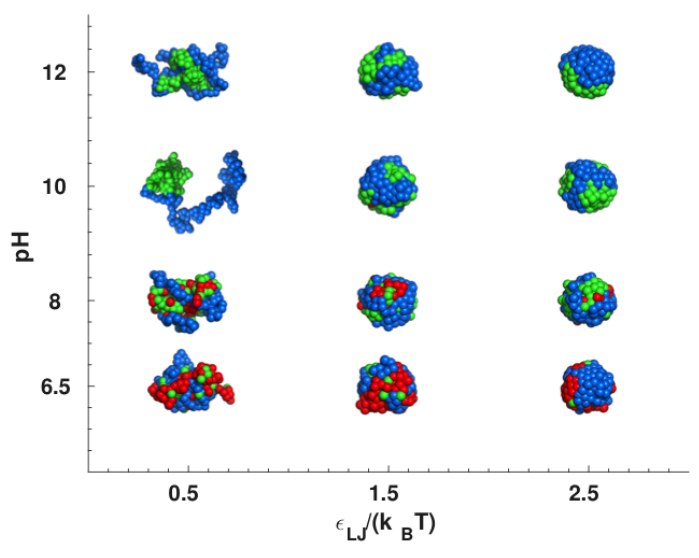

(a)

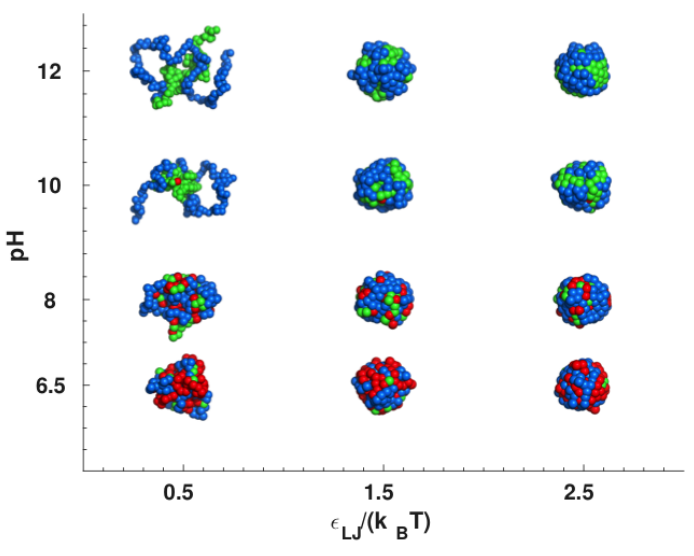

(b)

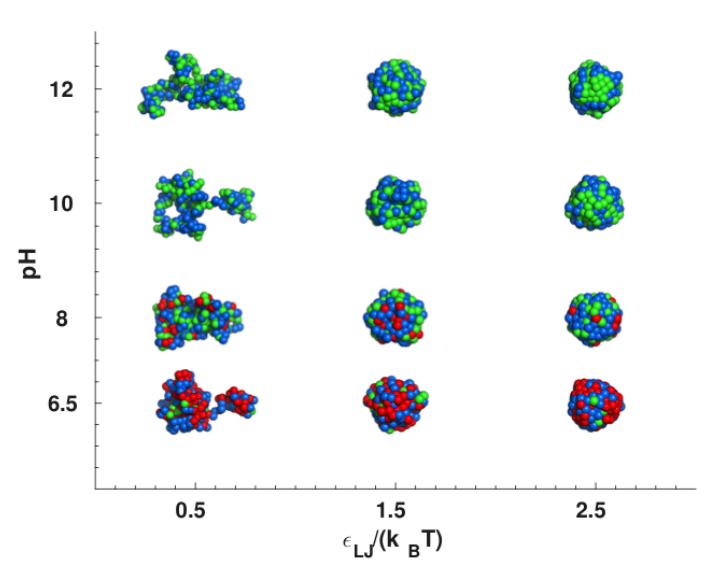

(c)

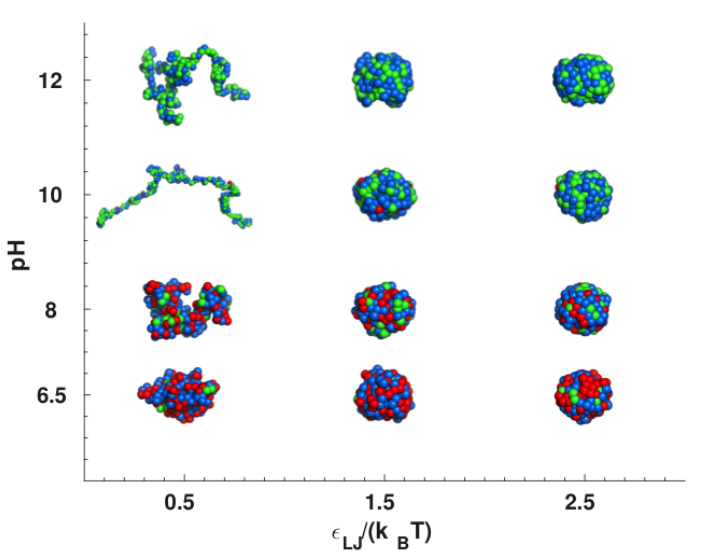

(d)

Figure 6: Same as in Fig. 3, but in the presence of salt (the Debye length $\lambda_{\mathrm{D}}=\sigma$ ). 


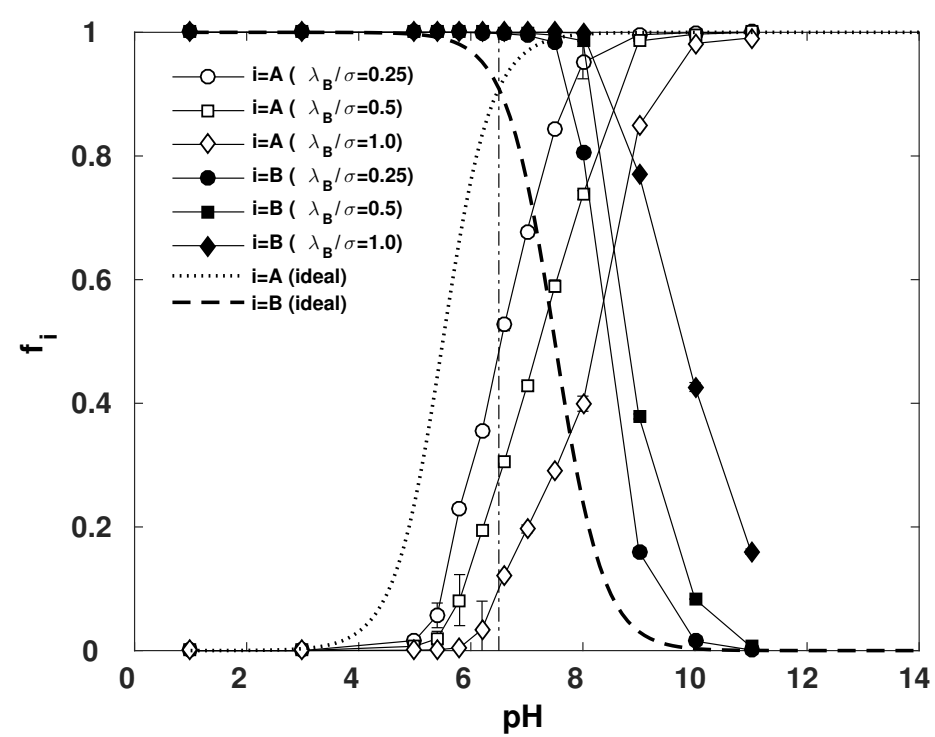

(a)

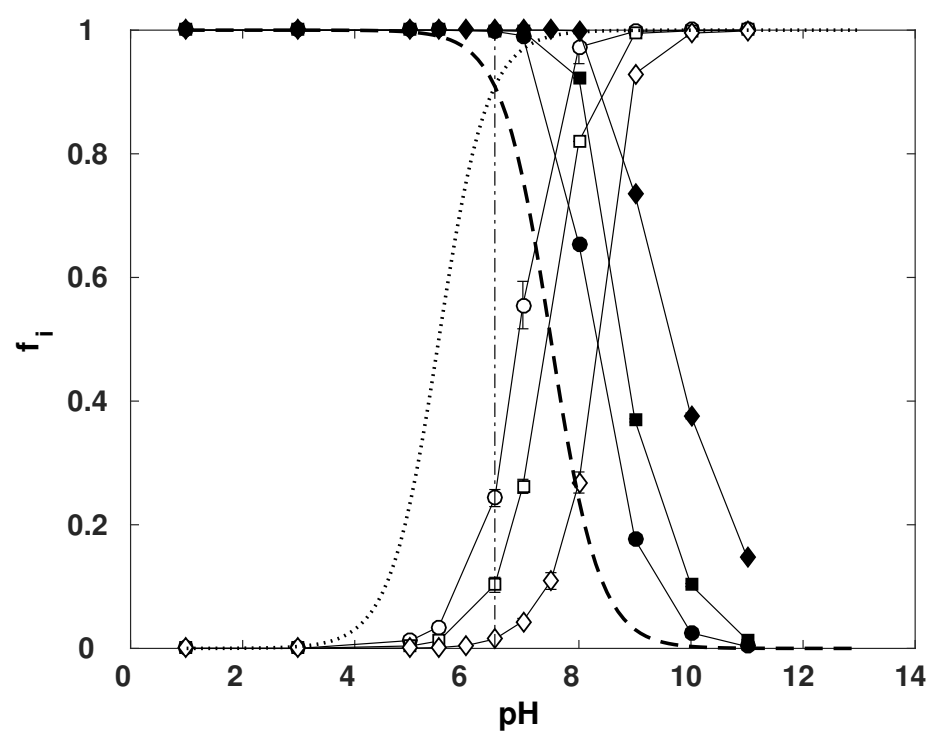

(b)

Figure 7: Titration curves of polyampholytes in the presence of a negatively charged polyelectrolyte at different values of the Bjerrum length $\lambda_{\mathrm{B}}$. Shown is the average charge fraction of monomers of type $\mathrm{i}\left(\mathrm{i}=\mathrm{A}\right.$ or $\mathrm{B}$ ) $f_{\mathrm{i}}$ as a function of solution $\mathrm{pH}$ : (a) diblock polyampholyte and (b) random polyampholyte. The values of $\mathrm{pK}_{0}^{\mathrm{A}}$ and $\mathrm{pK}_{0}^{\mathrm{B}}$ are 5.5 and 7.5 , respectively. 


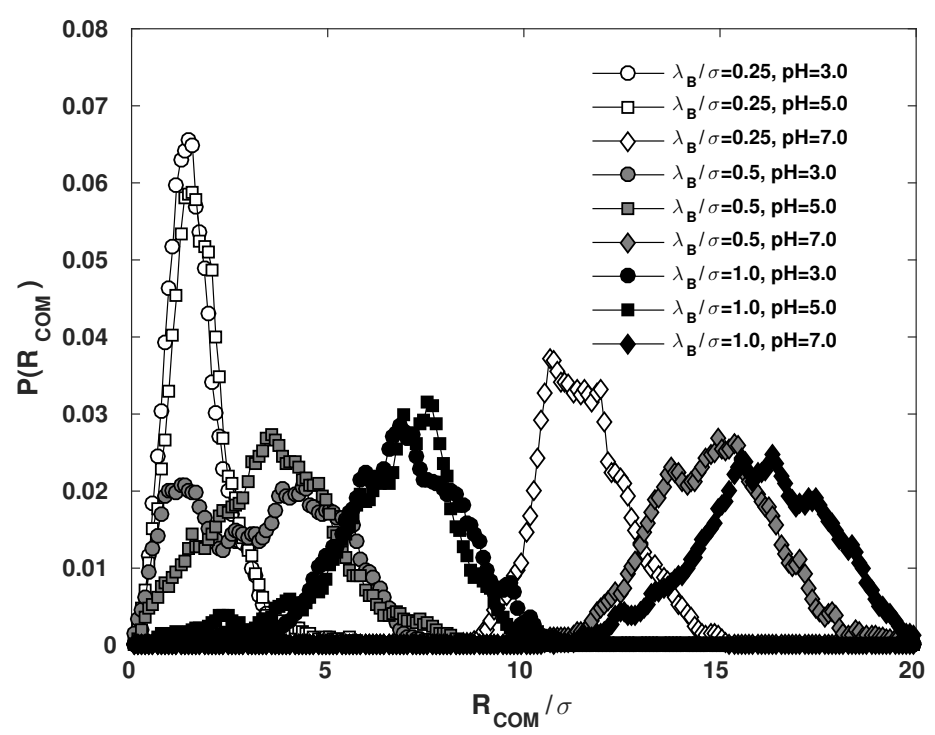

(a)

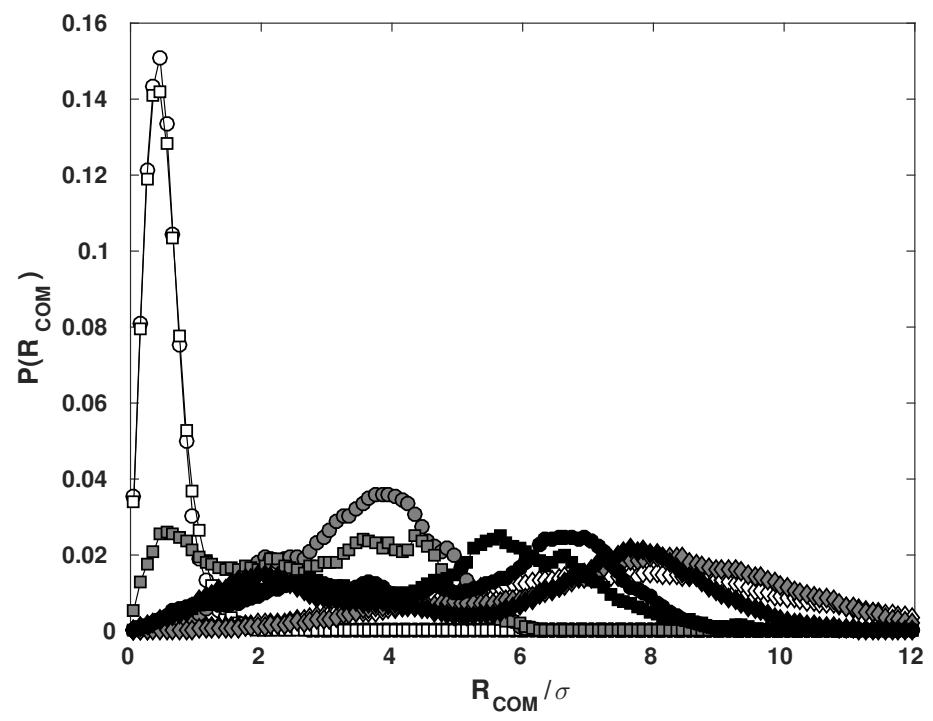

(b)

Figure 8: Probability distribution function $P\left(R_{\text {com }}\right)$ of the distance between the center of masses $R_{\text {com }}$ of polyelectrolyte and polyampholyte chains at different values of the solution pH: (a) diblock polyampholyte and (b) random polyampholyte. 


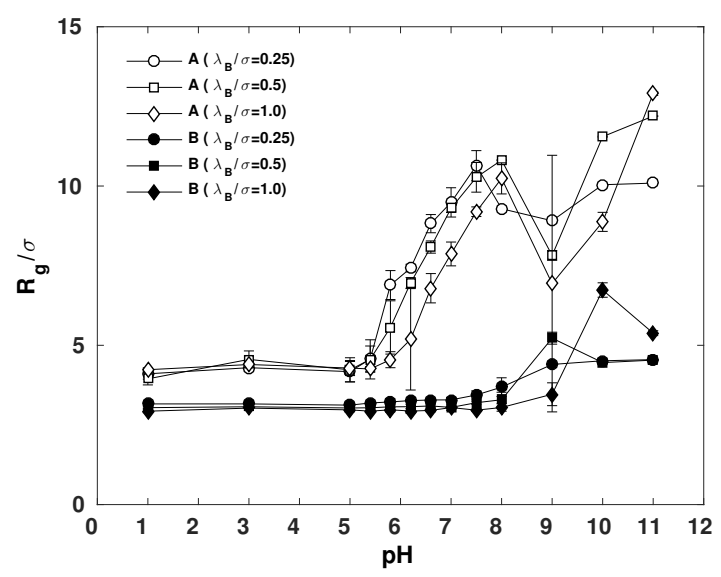

(a)

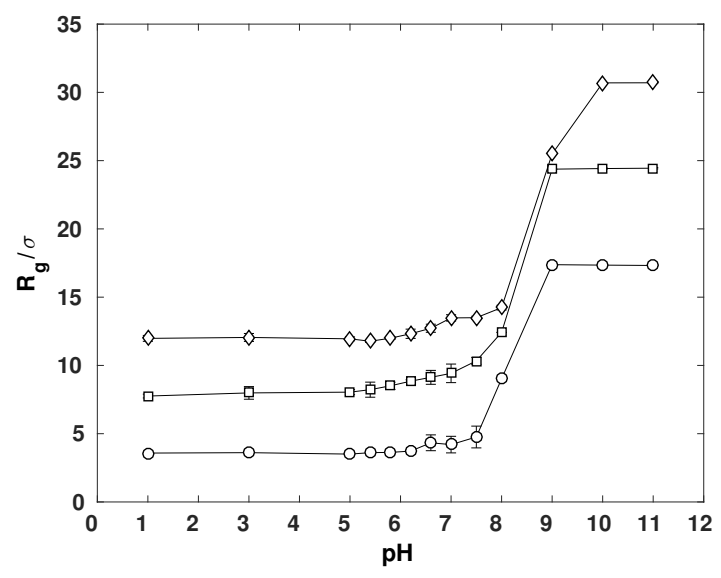

(b)

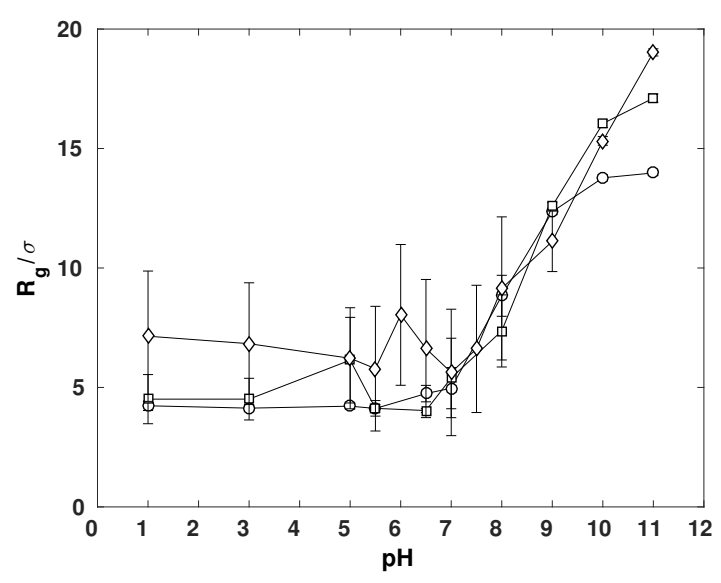

(c)

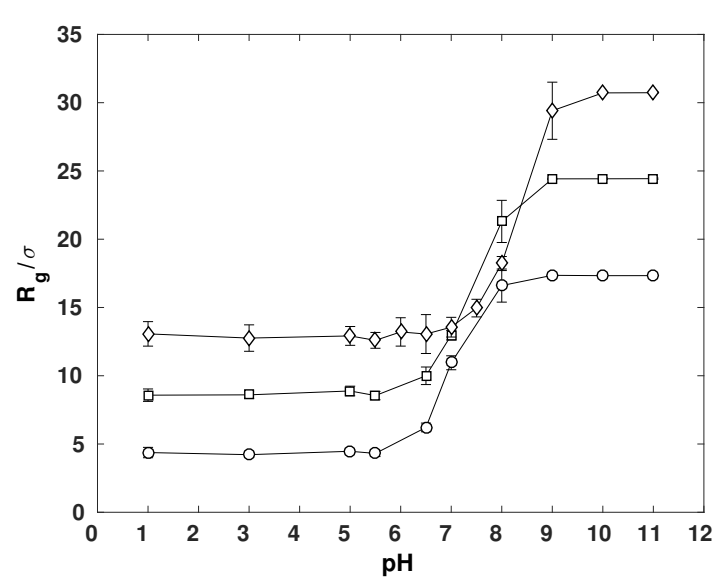

(d)

Figure 9: Root-mean-square radius of gyration $R_{\mathrm{g}}$ of polyelectrolyte-diblock (left panels) and polyelectrolyte-random (right panels) polyampholyte systems as a function of solution pH. Shown are the results of polyampholyte (top panels) and corresponding polyelectrolyte chains (bottom panels). Symbols are the same as given in Fig. 7. Note that the size of each block of the diblock polyampholyte is provided. 


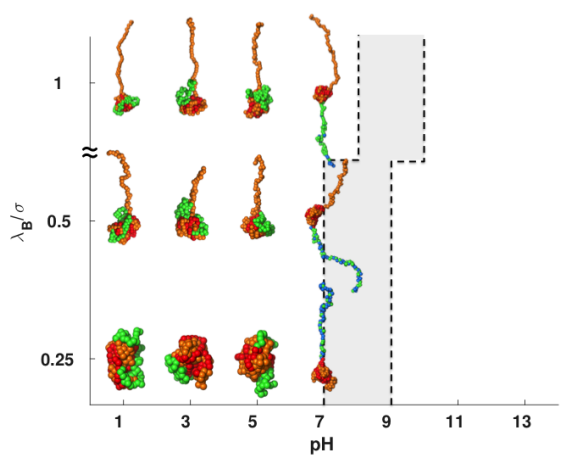

(a)

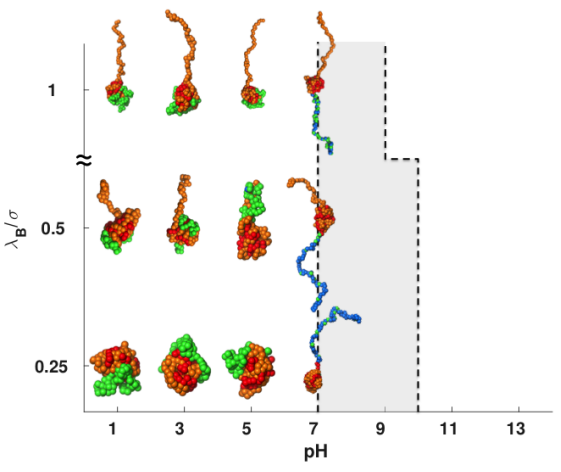

(b)

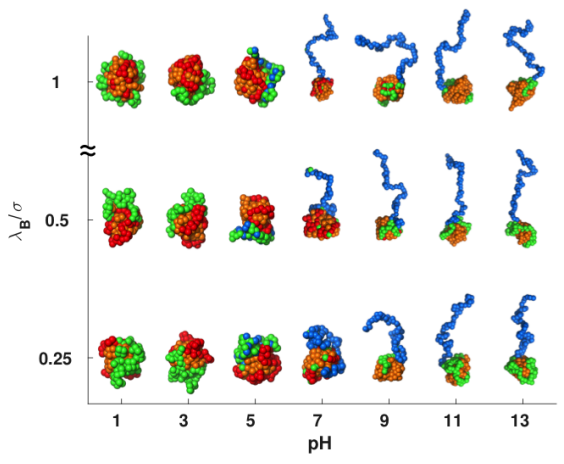

(c)

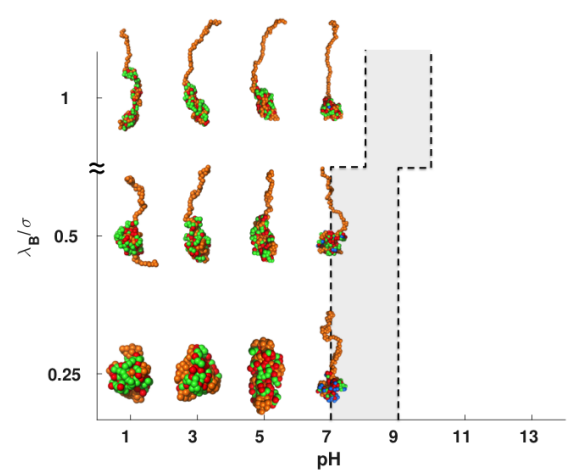

(d)

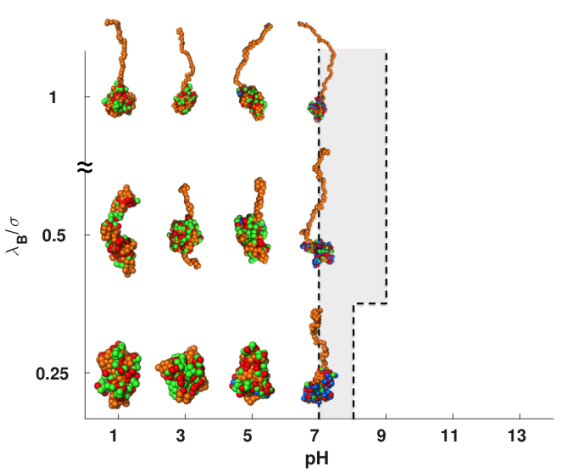

(e)

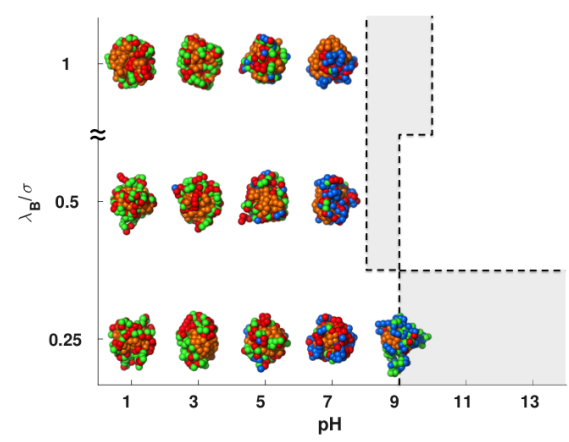

(f)

Figure 10: Typical conformations of polyelectrolyte-diblock (left panels) and polyelectrolyte-random (right panels) polyampholyte complexes in the $\mathrm{pH}-$ Bjerrum length $\lambda_{\mathrm{B}}$ plane without (top panels) and with added salt (the Debye length: $\lambda_{\mathrm{D}}=10 \sigma$ (middle panels) and $\lambda_{\mathrm{D}}=\sigma$ (bottom panels)). Color codes of snapshots are the same as in Fig. 3. In addition, negatively charged monomers of the polyelectrolyte are colored orange. The configurations are in the desorbed state for $\mathrm{pH}$ values above the coexistence region (grey). 


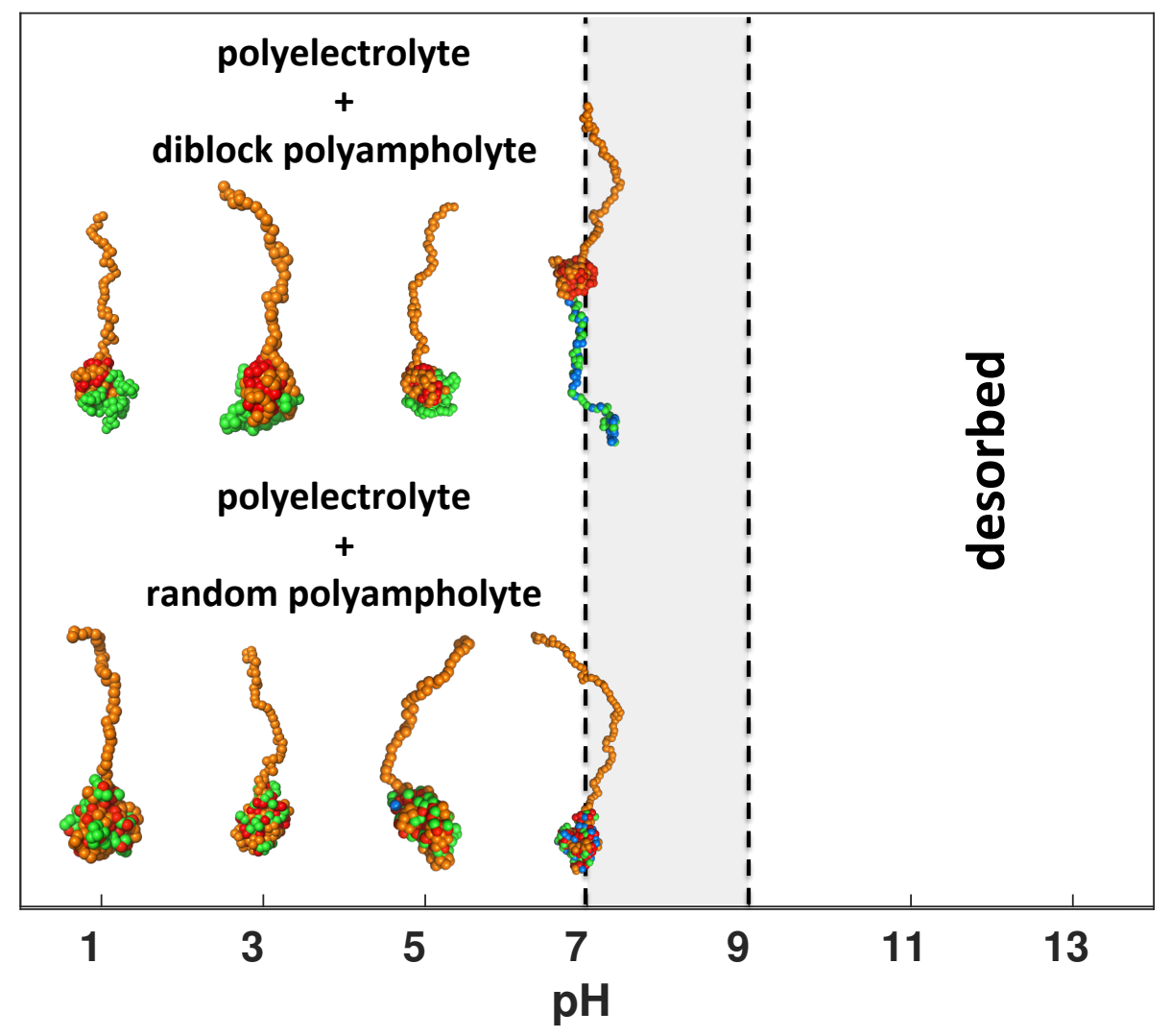

\section{TOC Graphic}

